\title{
MARIPOSAS DIURNAS ENDÉMICAS DE LA REGIÓN PALEÁRTICA OCCIDENTAL: PATRONES DE DISTRIBUCIÓN Y SU ANÁLISIS MEDIANTE PARSIMONIA (LEPIDOPTERA, PAPILIONOIDEA)
}

\author{
E. García-Barros*
}

\begin{abstract}
RESUMEN
La distribución de las mariposas endémicas del oeste de la Región Paleártica se emplea para producir una clasificación de las unidades de área mediante un coeficiente de asociación y mediante un análisis de parsimonia, y se comparan los resultados. Se demuestra que el procedimiento PAE-PCE propuesto por García-Barros et al. (2002) no garantiza la detección de todas las áreas delimitadas por endemismos de distribución exactamente coincidente. Sin embargo, ese procedimiento es eficaz si se aplica asociado a un método de búsqueda de cladogramas que proscriba la homoplasia, como el principio de compatibilidad. Empleado como método de clasificación, el análisis de parsimonia puede producir resultados ampliamente coincidentes con los de otros procedimientos de jerarquización. Convendría distinguir dos acepciones distintas del análisis de parsimonia aplicado a este tipo de datos, que representan interpretaciones estáticas de los cladogramas de áreas (sensu Rosen, 1988): el PAE en un sentido estricto (para detectar áreas de endemicidad, potencialmente solapadas y por tanto con múltiples soluciones), y la metodología cladística aplicada como método de agrupación (idealmente con una única solución, la de mayor parsimonia). Sobre la base de la fauna de mariposas, las áreas montañosas de la periferia del mediterráneo occidental presentan la mayor concentración de elementos endémicos, junto con las islas mediterráneas de mayor tamaño. la Península Ibérica muestra además el mayor número de áreas de endemicidad netamente diferenciadas. Los patrones de distribución de la fauna endémica parecen ajustarse, en líneas generales, a los del conjunto de la fauna de papilionoideos.
\end{abstract}

Palabras clave: Mariposas, Lepidoptera, Paleártico, biogeografía, parsimonia, endemismo, diversidad, PAE.

\section{ABSTRACT \\ Endemic butterflies of the Western Palaearctic: distribution patterns, and their analysis based on parsimony (Lepidoptera, Papilionoidea)}

The geographic distributions of the butterflies endemic to the Western Palaearctic are used to classify operative area units based on an index of association, and on parsimony analyses, and these results are compared. It is shown that the PAE-PCE method proposed by García-Barros et al. (2002) does not warrant detection of all subsets of area units characterised by endemic elements with identic distributions. However, satisfactory results can be achieved if Wager parsimony is subtituted by a method of cladogram construction that proscibes homoplasy, such as compatibility. When used as a classification procedure, the results of parsimony analysis can be broadly consistent with those derived from other procedures of hyerarchical classification. A distinction should be made between two different usages of parsimony, both of which represent 'static' (sensu Rosen, 1988) interpretations of area cladograms: PAE in a strict sense, aimed at detecting putative areas of endemicity. These are potentially overlapping entities, and hence the method may imply potentially multiple solutions. And, cladistic procedures applied as an agglomerative method (ideally with a single, most parsimonious solution). As far as the butterfly faunas are concered, the highest concentration of endemisms occur across the western circum-mediterranean mountain chains, and in the largest Mediterranean islands. The Iberian Peninsula is featured not just by a high concentration of endemic butterflies, but also by the coexistence of several distinc areas of endemism. Broad patterns derived from the endemic fauna fit well to those based on the whole butterfly fauna.

Key words: Butterflies, Lepidoptera, Palaearctic, biogeography, parsimony, endemism, diversity, PAE.

\footnotetext{
* Departamento de Biología (Zoología), Universidad Autónoma de Madrid, E-28049 Madrid, España. garcia.barros@uam.es
} 


\section{Introducción}

Una de las ideas dominantes en el concepto de área de endemicidad es la de demarcación geográfica más o menos limitada, caracterizada por las distribuciones aproximadamente congruentes de un conjunto de especies exclusivas de ella (Nelson \& Platnick, 1981; Major, 1988; pero ver, por ejemplo Harold \& Mooi, 1994; Posadas \& MirandaEsquivel, 1999; Espinosa et al., 2001). Es cierto que no existe unanimidad a propósito de la definición de un área de endemismo, ni de los criterios que debe reunir, lo que depende en buena parte de la definición de endemismo. Si bien estas áreas son frecuentemente consideradas como resultados de un proceso histórico, es prudente aceptar que, a priori, representan agrupaciones operativas, pendientes de interpretación, y que debieran entenderse como "áreas de endemicidad potenciales" (Hausdorf, 2002; Mast \& Nyffeler, 2003). Sea como fuere, la información asociada a ellas representa un complemento importante a la relacionada con los criterios de similitud, densidad, diversidad o riqueza de especies.

Dada la analogía entre área de endemicidad (caracterizada por especies exclusivas, sinapocorías) y grupo monofilético (caracterizado por sinapomorfías), los métodos basados en el principio de parsimonia proporcionan una manera de identificar estas áreas. Tales análisis, aplicados a la identificación de áreas de concentración de endemismos, se han usado bajo la denominación de $P A E$ (Parsimony Analysis of Endemicity: Morrone, 1994), Análisis de Simplicidad de Endemismos (Crisci et al., 1994), o PAD (Parsimony Analysis of Distributions: Trejo-Torres \& Ackerman, 2001). El método básico parte de la propuesta de Rosen (1988) de realizar cladogramas de áreas usando como caracteres las presencias de especies, mediante el principio de parsimonia. Requiere una matriz de presencias y ausencias de especies ( $u$ otros taxones) en unidades de área operativas. Las primeras se emplean como caracteres y las segundas como casos, fijando para comparación un grupo externo hipotético "vacío de especies" (Morrone, 1994; Morrone \& Crisci, 1995; Posadas y Miranda-Esquivel, 1999; Espinosa et al., 2001). El PAE se ha aplicado en diferentes regiones geográficas y variados grupos taxonómicos (Myers, 1991; Balletto, 1995; Da Silva \& Oren, 1996; Posadas et al., 1997; Geraads, 1998; Linder, 2001; Trejo-Torres \& Ackerman, 2001; Morrone \& Escalante, 2002: Rojas-Soto et al., 2003; y otros ejemplos en Llorente Bousquets \& Morrone, 2001).
En realidad, los análisis de parsimonia se han aplicado a matrices de datos binarios de distribución de tres maneras: 1) Como método biogeográfico para inferir relaciones históricas entre áreas, su propósito original (Rosen, 1988, y referencias en Morrone \& Crisci, 1995; Espinosa et al., 2002). Técnicamente, es posible incluir cierto tipo de información filogenética, o cuando menos taxonómica, si la filiación de las especies se codifica como presencia o ausencia de taxones supraespecíficos. Datos así pueden ser procesados como tales, o incluso en combinación con la información relativa a las especies (p. ej. Glasby \& Alvarez, 1999; Posadas \& Miranda-Esquivel, 1999). Pero la combinación de diferentes niveles taxonómicos, no convenientemente jerarquizados, produce resultados sin sentido claro, confundiendo la historia de las áreas implicadas con la de sus asociaciones ecológicas (Posadas \& Miranda-Esquivel, 1999). De hecho, se ha criticado seriamente la conveniencia de este procedimiento como método propio de la biogeografía histórica (Brooks \& van Veller, 2003), por lo que no se incidirá aquí en esta interpretación. Las otras dos acepciones o aplicaciones incluyen: 2) la delimitación o identificación de áreas de endemicidad, es decir, zonas caracterizadas por una acumulación de elementos faunísticos o florísticos exclusivos (1o que podríamos denominar PAE en un sentido estricto, o sensu Morrone, 1994). Y 3) como método de asociación entre unidades de área (interpretación estática de Rosen, 1988; por ejemplo Balletto, 1995), donde el principio de parsimonia es aplicado como pudiera serlo un índice de asociación o similitud clásico. Ambas interpretaciones guardan un marcado paralelismo, pero no son equivalentes, y en ellas se centra el presente estudio.

Como criterio de asociación, el principio de parsimonia proporciona un medio para clasificar $u$ organizar jerárquicamente conjuntos de unidades de área (aplicaciones análogas a la sinecología han sido sugeridas por Nel et al., 1998), al tiempo que permite designar especies características de las mismas. Dadas las propiedades del grupo externo, los patrones obtenidos dependen fundamentalmente de la congruencia entre las distribuciones observadas de las especies (más detalles en Geraads, 1998, Enghoff, 2000). En condiciones ideales, ofrece una única solución. Aplicado a la localización de áreas de endemicidad, el PAE permite detectar grupos de unidades de área operativas que estén respaldadas por al menos dos "sinapomorfías" (sinapocorías), es decir, especies exclusivas de esos subconjuntos de unidades de área (Morrone, 1994; Morrone \& Crisci, 1995). Esto significa, en la prác- 
tica, encontrar los subconjuntos de especies de distribución completamente coincidente. El principio de parsimonia representa una manera de obtener esos subconjuntos, que ciertamente podrían ser descubiertos por otros medios (por ejemplo análisis de compatibilidad: Meacham \& Estabrook, 1985). Presenta un problema: en tanto que se reconozca la potencial existencia de áreas de endemicidad superpuestas (Martín-Piera \& Sanmartín, 1999 y referencias allí citadas, aunque no hay consenso sobre este punto: Linder, 2001), la búsqueda de un único resultado con máxima parsimonia puede conducir a la pérdida de información. Si el poblamiento de especies endémicas de un área geográfica puede ser resultado de más de un evento histórico (hipótesis razonable en regiones de compleja historia biogeográfica), cabe más bien esperar múltiples soluciones potenciales. Esto diferencia este uso del PAE (para designar áreas de endemicidad a partir de conjuntos de unidades de área) de la acepción anterior (para clasificar conjuntos de tales unidades).

Con vistas a hallar las posibles soluciones múltiples mencionadas, García-Barros et al. (2002) han propuesto un procedimiento iterativo (PAE-PCE: PAE with Progressive Character Elimination). Requiere sucesivos análisis de parsimonia, tras cada uno de los cuales se desactivan los caracteres (especies, sinapocorías) que en ese punto sirvieron para identificar un área de concentración de endemismos, y se procesa nuevamente la matriz de datos, favoreciendo así la emergencia de agrupaciones de unidades de áreas alternativas a (no congruentes con) la más parsimoniosa. La eficacia de esta propuesta carece de comprobación empírica adicional, por lo que sería interesante aplicarlo a matrices de datos que manifiesten escasa congruencia entre áreas de distribución, pocas especies de amplia distribución y, en consecuencia, resultados basados en árboles de consenso de baja resolución. Esas características hacen adecuado al efecto el caso de las mariposas endémicas europeas, que se aborda en este trabajo. Luna Vega et al. (2000) han usado una metodología similar a la descrita. Si bien encaminado al establecimiento de pistas generalizadas (generalized tracks, trazos generalizados, p. ej.: Brundin, 1988), su método resulta ser idéntico al PAE-PCE antes descrito, salvo por la proscripción de la homoplasia para conseguir los efectos del método de compatibilidad. Este detalle podría suponer ventajas sobre el uso de la parsimonia de Wagner en los análisis de PAE, así que se incorporó esta variante con el fin de comparar los resultados.

La reciente aparición de un atlas de la distribución europea de las mariposas diurnas (Kudrna,
2002) proporciona un pretexto para comprobar los extremos indicados. En comparación con muchos otros grupos de animales invertebrados, estos insectos son buenos candidatos para el trabajo biogeográfico, ya que por diversos motivos su distribución geográfica se conoce con razonable aproximación. Además de trabajos pioneros (Kostrowicki, 1969), la fauna europea de estos lepidópteros ha sido objeto de análisis para su tipificación, sectorización, y determinación de patrones relativos a la distribución de su diversidad de especies (Balletto, 1995; Dennis et al., 1991, 1995a, 1995b, 1998). Si bien ningún trabajo anterior, a esta escala geográfica, ha empleado datos de distribución con referencia geográfica precisa, no se profundizará aquí en una disección detallada de la información. No obstante, un estudio descriptivo preliminar de los datos de distribución empleados es interesante desde el punto de vista de la biogeografía de estos insectos, al igual que algunas precisiones relativas al sector occidental del área interesada, que incluye el ámbito ibérico.

En consecuencia, las líneas que siguen pretenden, en primer lugar, abordar una sectorización del área paleártica occidental por su fauna endémica de mariposas diurnas mediante el principio de parsimonia, interpretando de un modo general los resultados. Incidentalmente, esto permite poner a prueba el método PAE-PCE para detectar áreas de endemicidad superpuestas y, con ello, ilustrar algunas dificultades de la aplicación del principio de parsimonia como criterio de asociación de unidades de área.

\section{Material y métodos}

\section{1) Área de estudio y fuentes}

El área de estudio quedó definida por la cubierta con cierta fiabilidad por los datos faunísticos europeos recogidos por Kudrna (2002), es decir Europa desde las Islas Británicas hasta aproximadamente la altura del Mar Negro. La zona europea oriental muestra un evidente déficit de información, por lo que fue excluida. Fue posible complementar estos datos para el oeste de Turquía gracias al trabajo de Hesselbarth et al. (1995), y en gran parte para el norte de África. Para esto último se empleó la guía de Tolman \& Lewington (1997) cotejada con mapas de distribución anteriores (Higgins \& Riley, 1980; Higgins \& Hargraves, 1983) y complementada, donde fue posible, por datos de Tennent (1996). La información relativa a este área deriva, por lo tanto, de mapas de manchas de distribución, y se desconoce su nivel de preci- 
sión, defecto que probablemente quede en parte compensado por el amplio tamaño de malla empleado. En algunos casos fue posible, puntualmente, actualizar o corregir la información meramente bibliográfica. Se adoptó una rejilla rectangular con unidades de un grado y medio de latitud por tres grados de longitud, tres veces mayor que la original del Atlas de Kudrna (2002), con intención de limar en cierta medida la posible heterogeneidad de la intensidad de muestreo. Para la denominación de las unidades de área se ha empleado un sistema arbitrario de letras (abcisas) y números (ordenadas) (Figuras 1 y siguientes).

\section{2) SELECCión taxonómica}

Los datos consisten en la presencia (1) o ausencia (0) de especies o subespecies de Papilionoidea (familias Papilionidae, Pieridae, Lycaenidae y Nymphalidae) endémicas del área de estudio (en lo sucesivo denominadas simplemente "endemismos"), es decir, presentes exclusivamente dentro del área considerada. Este criterio se relajó ligeramente para incluir algunas especies que alcanzan el centro $u$ oriente de Turquía, concretamente Lycaena ottomana Lefèbvre, 1830, Polyommatus escheri (Hübner, 1823); y Aricia cramera (Eschscholtz, 1821), que está presente en las Islas Canarias, además de en el área continental europea $\mathrm{y}$ norteafricana. La combinación de especies y subespecies tiene tres justificaciones: seleccionar taxones de distribución tan limitada y definida como fuera posible, con máxima garantía de identidad genética de los endemismos; subsanar parcialmente el problema creado por la subestimación de elementos orientales (ver discusión). Finalmente, evitar una justificación pormenorizada de la selección taxonómica en comparación con las especies reconocidas por Kudrna (2002), que no es aquí pertinente. En el caso de las subespecies, no obstante, la muestra se limitó a aquellas que cuentan con un cierto reconocimiento por parte de los taxónomos, y presentan áreas de distribución nítidamente separadas y definidas. Ninguno de los criterios adoptados representa opinión alguna por parte del autor sobre el estatus taxonómico de cualquiera de los endemismos tratados. La matriz de datos, de 245 unidades de área y 196 endemismos, puede verse en el Apéndice.

\section{3) ANÁLISIS PROSPECTIVO}

Se realizó un primer estudio de los datos con pretensión principalmente descriptiva, calculando la asociación entre las unidades de área mediante el índice de Jaccard, que se empleó para construir un dendrograma por el método de ligamiento promedio no ponderado (UPGMA) mediante las opciones de clasificación de conglomerados jerárquicos del paquete estadístico SPSS (SPSS Inc., 2001). El coeficiente de Jaccard se adoptó para facilitar la comparación con los trabajos de Dennis et al. (1991-1998), en los que se ha usado con profusión y que, al igual que el principio de parsimonia, no valora las ausencias compartidas. La matriz de datos original se sometió de modo prospectivo a un análisis factorial con extracción de factores por el método de componentes principales, sin rotación (mediante STATISTICA: Statsoft, 1996). El número de factores extraidos se determinó de modo intuitivo (ver resultados).

\section{4) Métodos de PAE}

Para el análisis mediante parsimonia de Wagner se empleó en primer lugar la combinación de los programas Winclada y Nona (Goloboff, 1993b; Nixon, 1999a). Para cada análisis independiente se estableció un número de 500 réplicas, 100 árboles iniciales por réplica, selección de 50 árboles (hold), y el procedimiento reiterativo de bisección y reconexión múltiple (Multiple TBR $+T B R$ ). Se empleó, además, el procedimiento de racthet (Nixon, 1999b). En cada caso se retuvo el árbol de consenso estricto de la series de cladogramas de menor longitud, y se calculó el índice de bootstrap (500 réplicas) para obtener una idea del soporte de las áreas de endemicidad identificadas. La ditribución de los endemismos se optimizó en el árbol de consenso, detectando los libres de homoplasia, y las áreas caracterizadas por al menos dos de ellos. Estos "caracteres" fueron desactivados, y la matriz fue reanalizada (con las especificaciones ya descritas) para intentar descubrir nuevas áreas de concentración de endemismos que fuesen incompatibles con las obtenidas en el primer análisis. Estos pasos se repitieron de modo cíclico hasta que no aparecieron nuevos conjuntos de unidades de áreas definidos por sinapocorías libres de homoplasia. De modo independiente, se siguió el procedimiento descrito por Luna Vega et al. (2000), procesando la misma matriz de datos mediante el programa PAUP (Swofford, 2002) con el ajuste de Goloboff (1993a) de valor de $\mathrm{K}=0$ (comandos PSET; $G O L O B O F F=Y E S ; G K=0$ ), que permite obtener series de cladogramas basados en los mayores subconjuntos de caracteres no homoplásticos. El sistema de eliminación progresiva de especies y reanálisis se efectuó a partir de los primeros resultados, del modo ya descrito (ver también Luna Vega et al., 2000). 


\section{Resultados}

El tamaño medio del área de distribución de los endemismos tratados no es exageradamente pequeño, con un promedio de 11,63 cuadrículas por especie $($ d.e. $=14,45)$, pero hay una importante proporción de endemismos de distribución puntual. El número de endemismos por unidad de área varía ampliamente (media $=9,73$, d.e. $=12,97)$, con una notable concentración circummediterránea, más marcada en la Península Ibérica y el entorno Alpino. Estas últimas parecen acumular una mayor proporción de endemismos de limitada distribución. Un resumen de esta situación se ofrece en la Figura 1.

Utilizando una escala arbitraria para medir la latitud y longitud ( 1 a $n$, dando el valor 1 a la cuadrícula más meridional y occidental), se constataron correlaciones significativas, si bien modera- das, entre la densidad de endemismos y la latitud y longitud (respectivamente $\mathrm{r}=-0,32$ y $\mathrm{r}=-0,37$; ambas $\mathrm{p}<0,0001)$. Incidentalmente, el tamaño promedio del área de distribución de los endemismos mostró una correlación estadísticamente significativa con la latitud $(\mathrm{r}=0,339 ; \mathrm{p}<0,0001)$, pero no con la longitud $(\mathrm{r}=0,06 ; \mathrm{p}=0,36)$. La Figura 2 muestra que esta relación es, probablemente, compleja: la extensión promedio del área de distribución parece alcanzar valores mínimos en los endemismos meridionales y septentrionales, y máximos dentro de un rango latitudinal que corresponde aproximadamente a la franja comprendida entre $\operatorname{los} 48^{\circ} \mathrm{N}$ y $\operatorname{los} 65^{\circ} \mathrm{N}$.

En el análisis factorial, los seis primeros factores permitieron explicar un $62,33 \%$ de la variación en los datos. Un examen del gráfico de pendientes del peso de estos factores sugería un número de
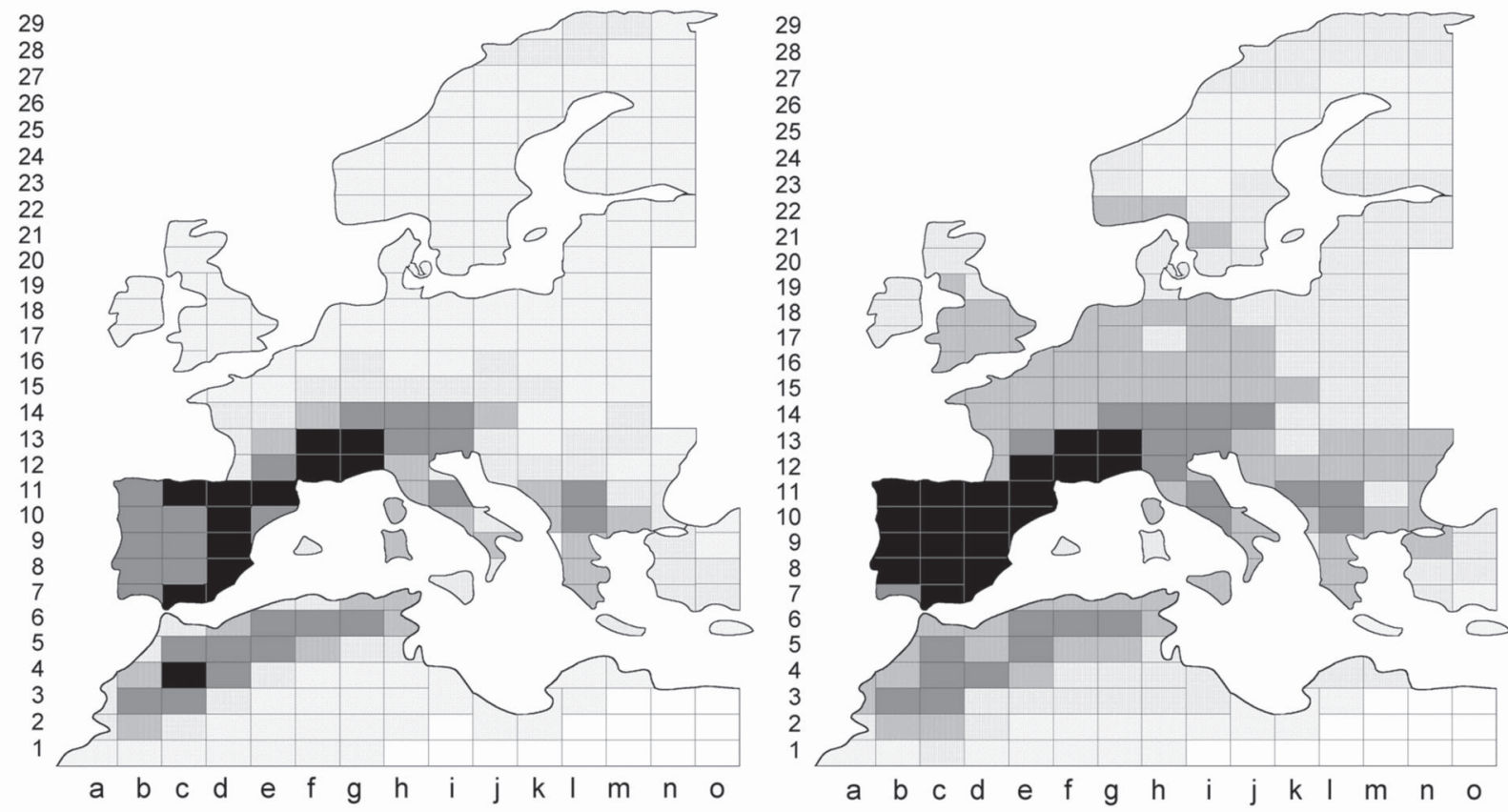

Fig. 1.- Izquierda, densidad de especies y subespecies endémicas en el área considerada. Los niveles de saturación corresponden respectivamente, de blanco a negro, a la escala: (1 a 4 endemismos), (5 a 10), (11 a 20), (21 a 40), y (más de 40). Derecha, originalidad acumulada de la fauna, calculada como el sumatorio de la rareza relativa de los endemismos de cada cuadrícula. Se tomó como índice de rareza el valor inverso del número de unidades de área ocupadas por cada especie, y la escala es relativa al valor máximo. Las áreas más oscuras representan los valores más altos.

Fig. 1.- Left, densities of species and subspecies endemic to the study area. The levels of saturation (increasing darkness) follow the scale: (1 to 4 endemisms), (5 to 10), (11 to 20), (21 to 40), and (more than 40). Right, cumulative originality of the endemic butterfly fauna, calculated as the sums of the relative rarities of the endemisms present in each square. Rarity was estimated as the inverse of the number of area units occupied by each species, rescaled as proportional to the maximum value. Darkest areas represent the highest values. 

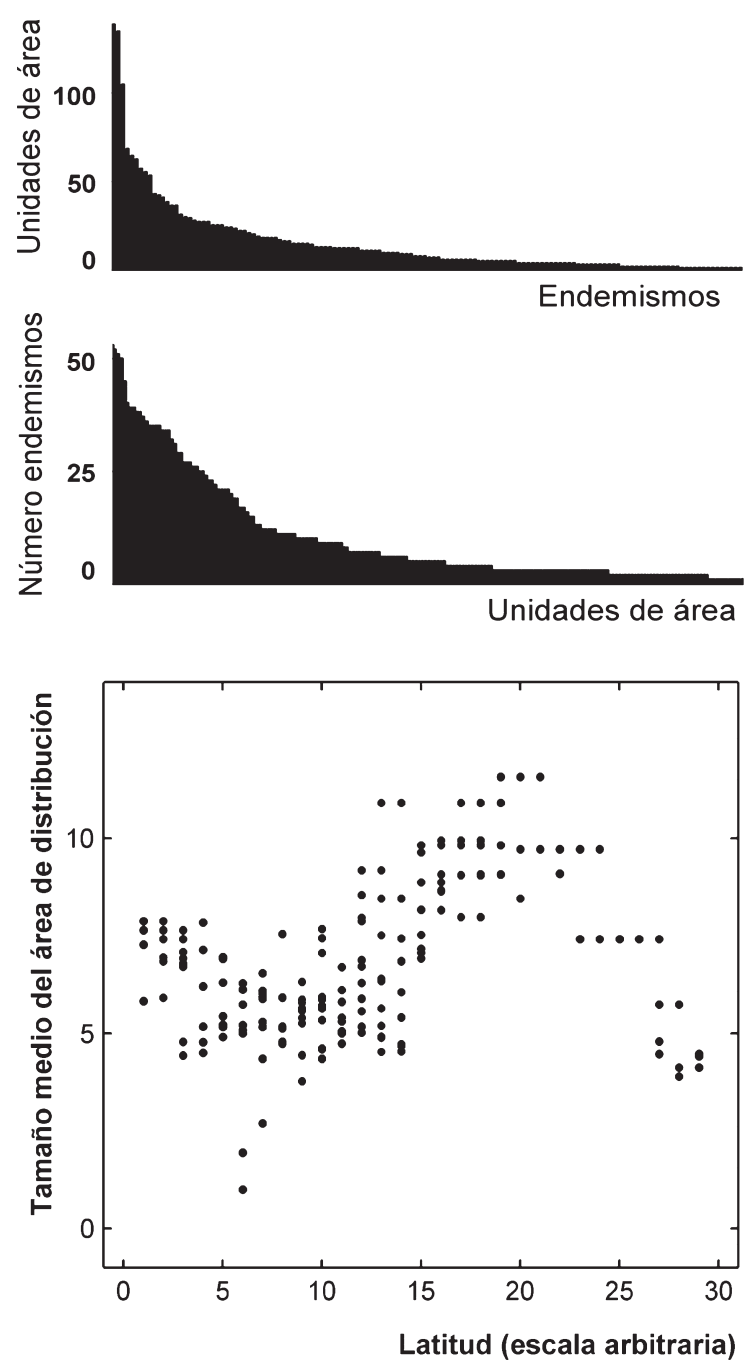

Fig. 2.- Características de la distribución de las mariposas endémicas en la región Paleártica occidental. Arriba, distribución de las frecuencias del número de unidades de área por taxón, y del número de taxones por unidad de área. Debajo, relación entre el tamaño medio del área de distribución de los endemismos con la latitud, por unidades de área $(\mathrm{r}=0,34$; $\mathrm{p}<0,0001)$. El tamaño de las áreas de distribución se midió como la raíz cuadrada del número de unidades de área ocupado por cada endemismo.

Fig. 2.- Main features of the distribution of endemic butterflies in the western Palaearctic. Above, frequency distribution of the number of area units per taxon, and number of taxa per area unit. Below, relationship between the average size of distribution area of endemisms, and latitude (data averaged by operative area units $)(r=0.34 ; p<0.0001)$. The size of geographic distributions was estimated as the square root of the number of quadrats occupied by each endemic taxon. cuatro $(53,59 \%$ de la varianza). En la Figura 3 se presenta, simplificado, un resumen de la relación de las unidades de área con respecto a los tres primeros factores (responsables del 48,58\% de la variación). Se han usado para ello los valores de las cargas factoriales para cada variable, que vienen a indicar la importancia de cada factor en la puntuación de cada variable inicial dentro del conjunto ( $\mathrm{p}$. ej. Catena et al., 2003). Estos parecen relacionados, por este orden, con un gradiente de diversidad y rareza, y la distribución de endemismos septentrionales y meridionales, por lo que la estructura tiende a pivotar en torno a un eje aparentemente caracterizado por la dominancia de especies de amplia distribución. Los resultados de la agrupación basados en el índice de Jaccard se resumen en las Figuras 4 y 5 ; dado el número de rectángulos analizado (245), no se ofrece un dendrograma completo. No se ha intentado comprobar el nivel de significación estadística de estas agrupaciones, si bien una serie de subconjuntos asociados a un nivel de $\mathrm{J}=0,4$ o superior admiten una explicación intuitiva senci1la (Figura 4). Detalles pertinentes a la Península Ibérica se muestran en la Figura 6.

El primer análisis mediante parsimonia resultó en un elevado número de árboles ( $>1000)$, con longitud de 616 pasos ( $\mathrm{ci}=31, \mathrm{ri}=80$ ), que produjeron un árbol de consenso estricto de 851 pasos (ci=23, $\mathrm{ri}=69$ ). El consenso presentó una politomía basal en la que se insertan un número de cuadrículas juntamente con algunos clados. Los últimos (resumidos en la Figura 6) arrojaron índices de bootstrap superiores a 0,66 , pero rara vez más elevados que 0,85 . Las áreas potenciales de endemicidad mejor apoyadas desde este punto de vista, de acuerdo con la notación empleada en la Figura 6 y Tabla 1, fueron la II (valor de bootstrap= 100), VI $(0,99)$, y III $(0,92)$, las tres dentro de los límites ibéricos. En total, se detectaron diez áreas de endemicidad definidas por al menos dos especies no presentes en ningún otro lugar (Figura 6, Tabla 1), pero sólo la mitad de ellas constaban de más de una unidad de área. Además, otro número de agrupaciones congruentes con las anteriores, pero definidas por una única especie. La estructura interna del clado que define la Península Ibérica se detalla en la Figura 7.

La aplicación del PAE-PCE, por eliminación de las especies características y nuevo análisis se repitió hasta diez veces. Se obtuvieron árboles de consenso cada vez menos consistentes, pero ninguna nueva área caracterizada por dos o más especies exclusivas. Una nueva serie de repeticiones, con eliminación de las especies características en el primer análisis, pero en diferentes órdenes, no arrojó 

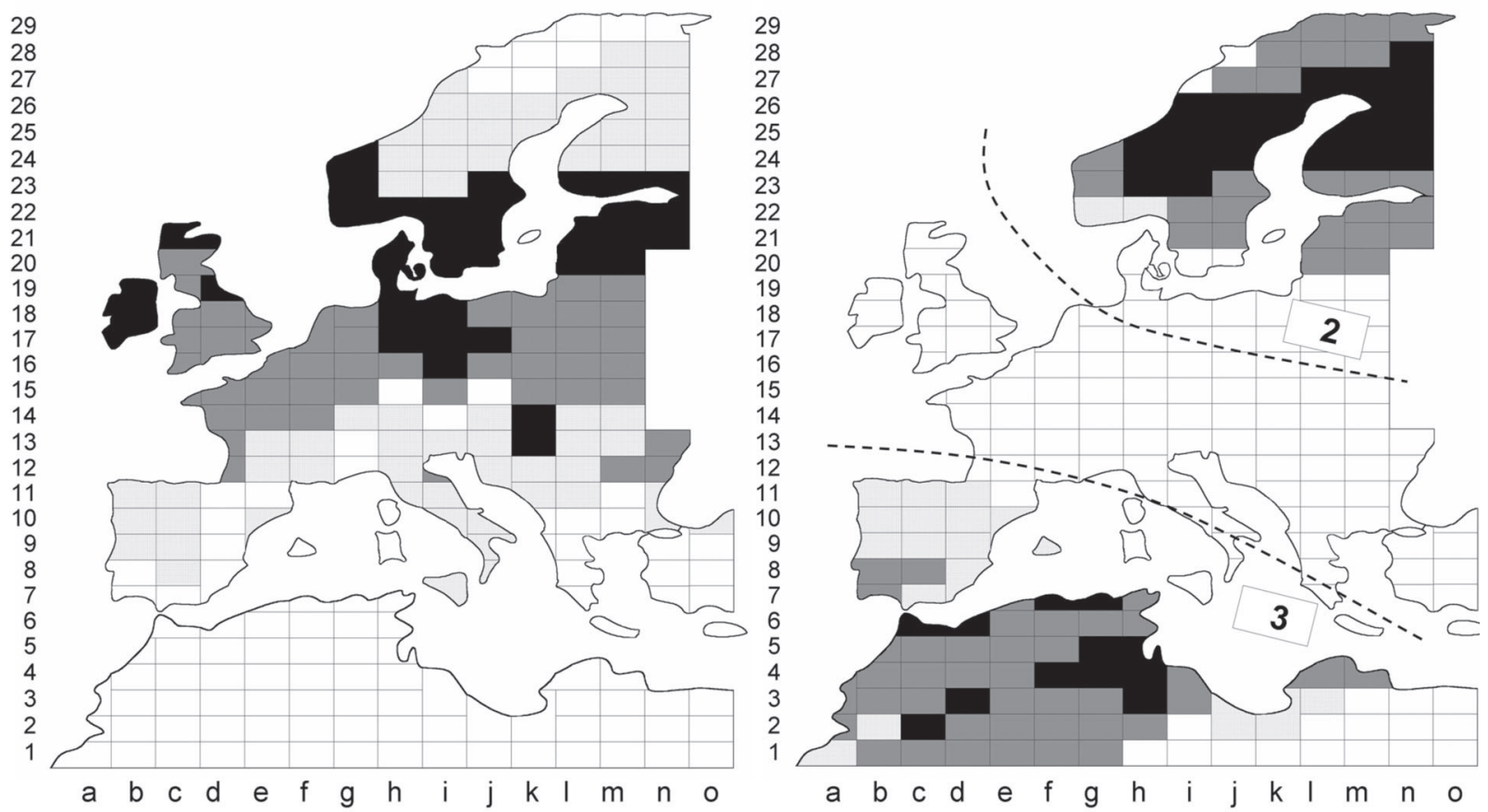

Fig. 3.- Resumen de la distribución entre las unidades de área de las cargas factoriales de los tres primeros factores extraidos (análisis factorial de los datos). Los valores relativos al primer factor se representan en el mapa de la izquierda. El mapa de la derecha resume, combinados en el mismo gráfico, los valores relativos a los factores 2 y 3 . Los niveles de saturación (blanco a negro) corresponden respectivamente a los rangos de valores $(0,00$ a 0,25$),(0,26$ a 0,50$),(0,51$ a 0,75$)$, y $(0,76-0,99)$, sean estos positivos o negativos según el factor.

Fig. 3. - Summary of the distribution across the study area of the factor loadings from the first three factors extracted (factor analysis). The values relative to the first factor extracted are presented in the left, map, and values from factors 2 and 3 are presented on the right map. Progressively increasing levels of saturation (darkness) represent highest absolute scores (either negative or positive), following the scale: $(0,00$ a 0,25$),(0,26$ a 0,50$),(0,51$ a 0,75$),(0,76-0,99)$.

ningún otro resultado. Para establecer un contraste, se calcularon a mano las posibles áreas de endemismo adicionales, por un procedimiento de tabulación manual: en la matriz de similitudes entre las cuadrículas (valores de Jaccard, si bien una matriz cuadrada de coeficientes de correlación podría servir para el caso), se identificaron los valores de semejanza absoluta $(J=1,00)$. Descartando los valores unitarios de la diagonal de la matriz, se procedió a trazar a mano los subconjuntos de áreas con faunas exactamente coincidentes no hallados en los cladogramas. Se encontraron tres áreas de endemicidad adicionales que aproximadamente corresponden, respectivamente, a la mitad norteña de Iberia, los Alpes septentrionales y occidentales, Jura y parte del Valle del Rin, y Alpes meridionales (regiones XI a XII: Tabla 1).

El procesado de los datos restringiendo la homoplasia mediante PAUP y con eliminación pro- gresiva de caracteres resultó notablemente más eficiente que la parsimonia de Wagner. La primera prueba arrojó un conjunto de cladogramas $(n>1000)$ de 673 pasos $(\mathrm{ci}=0,29, \mathrm{ri}=0,77)$ cuyo consenso estricto permitió encontrar las regiones IX y XII. La segunda y tercera repeticiones detectaron las regiones XI y XIII respectivamente.

\section{Discusión y conclusiones}

Aunque no se han realizado anteriormente estudios biogeográficos detallados sobre mariposas referidos a unidades de área uniformes y de tamaño relativamente pequeño en esta región, los datos empleados distan mucho de ser perfectos ni homogéneos (Kudrna, 2002). Por ello, y dado que hay cierto número de resultados anteriores basados en faunas de localidades o áreas típicas o 


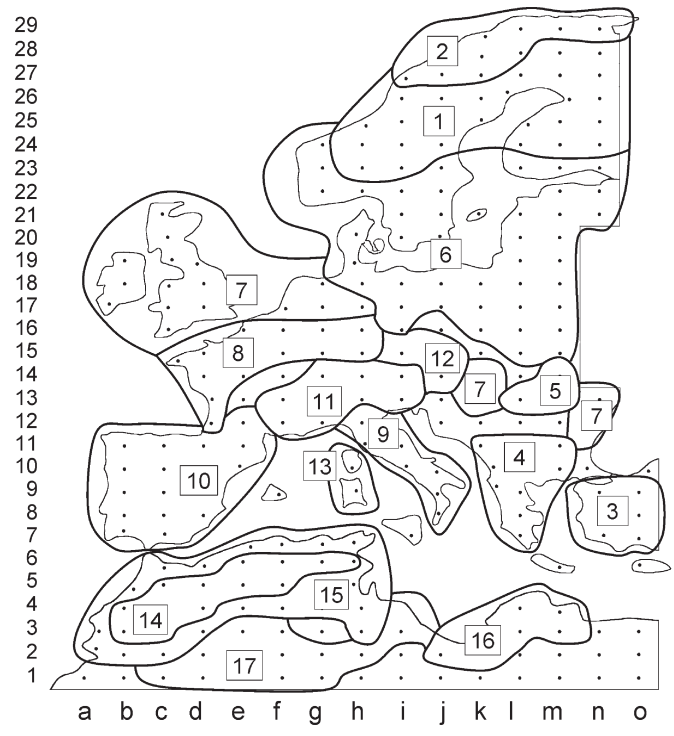

Fig. 4. - Agrupaciones de unidades de áreas obtenidas mediante la aglomeración (UPGMA) basada en el índice de asociación de Jaccard. Se representan los grupos unidos a un nivel igual o superior a 0,35; para mayor claridad, se omite la estructura interna de estos aglomerados. La numeración de las áreas es arbitraria (1 a 17), y corresponde con la empleada en la Figura 5.

Fig. 4.- Results of clustering area units following UPGMA method and using Jaccard's index as a measure of association. Only clusters joining at values equal or higher than 0.35 are presented (for clarity, the internal structure of these agglomerates is omitted). Labelling of sets of land quadrats (1 to 17) corresponds to that used in Figure 5.

representativas (Dennis et al., 1991-1998) tiene cierto interés comparar los patrones generales basados exclusivamente en elementos endémicos y una malla geográfica regular, aún considerando ciertas limitaciones. Es difícil, por ejemplo, determinar en qué medida la inclusión de taxones infraespecíficos afecta a los presentes resultados más allá de los efectos buscados (área de distribución promedio reducida, incremento de la información por taxón). Más importante, la exclusión de especies y subespecies que se extienden hacia el Este más allá del área considerada (y por definición, no son endémicas de la misma) puede ser responsable de un sesgo occidental importante. Concretamente, la fauna anatólica (área de Turquía, norte de Irán, Irak, etc.) es casi por completo ignorada en este trabajo. Como consecuencia de la situación marginal de esa zona dentro del marco geográfico escogido, diversos elementos faunísticos originales (p. ej. Balletto, 1995;

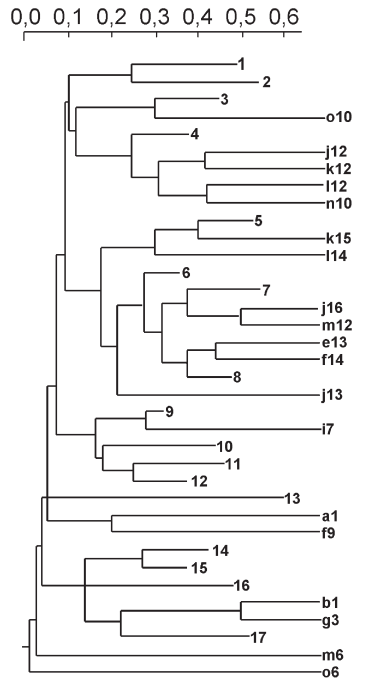

Fig. 5.- Dendrograma basado en el índice de asociación de Jaccard, mostrando la asociación entre los grupos mostrados en la Figura 4, y con otras cuadrículas no incluidas allí. Los números (1 a 17) corresponden a las áreas delimitadas en la Figura 4. Su posición con respecto a la escala de asociación indica la base del subárbol correspondiente a las cuadrículas implicadas, que no se presenta en detalle. Las combinaciones de letras y números representan unidades de área singulares.

Fig. 5.- Dendrogram based on Jaccard values, showing the association among the sets of squares shown in Figure 4 and other squares not shown therein. Labels (1 to 17) correspond to those used in Figure 4. The internal structure of the branches relating the units within the areas 1 to 17 are not shown in detail. The combinations of letters and numerals represent single area quadrats.

Hesselbarth et al., 1995; Tolman \& Lewington, 1997), no han sido contabilizados como endémicos del área en estudio. Un problema semejante interesa la fauna de mariposas subártica o circumboreal; ésta se caracteriza por un número de especies exclusivas originales, pero de muy amplia distribución longitudinal (p. ej. Kostrowicki, 1969), por lo que tampoco cumplen la condición de "endemismo" adoptada aquí. Con esto en mente, merece la pena considerar tres aspectos: 1) los resultados de aplicar el principio de parsimonia como método para la detección de áreas de concentración de endemismos, 2) su capacidad como método de clasificación por aglomeración, $3)$ los resultados más relevantes relacionados con de la clasificación de las unidades de área por sus endemismos en el área de estudio, con breve mención al área ibérica y balear. 


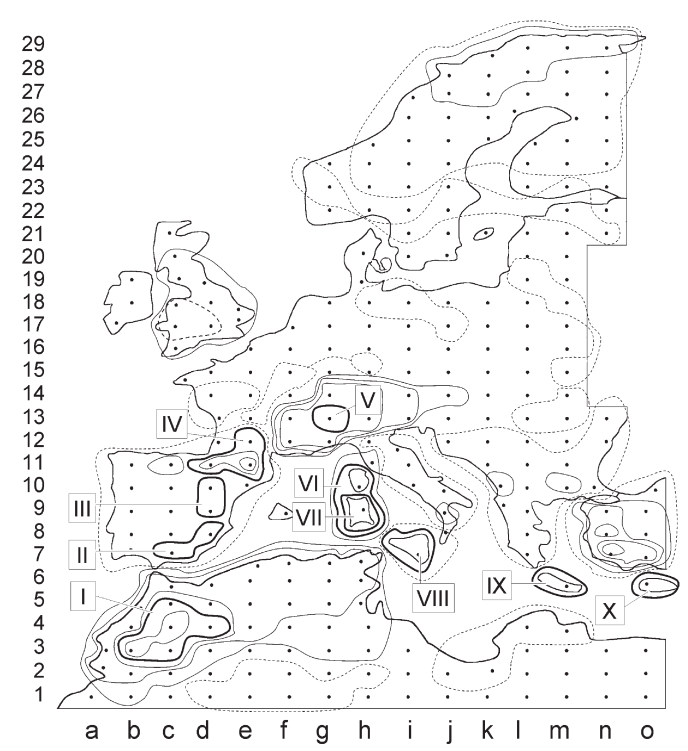

Fig. 6.- Áreas de endemicidad determinadas mediante el análisis de parsimonia de Wagner. Las agrupaciones de unidades de área caracterizadas por al menos dos endemismos (I a X) se señalan con línea gruesa; las líneas delgadas corresponden a la distribución de especies o subespecies que son congruentes con las áreas de endemicidad. Las líneas de trazos, a grupos de cuadriculas representados en el árbol de consenso y con un índice de bootstrap superior a 66, pero no caracterizadas por especies exclusivas. Detalles en la Tabla 1. Nótese que tres áreas de endemicidad (XI, XII, XIII) obtenidas por otros métodos no están representadas en la figura (ver detalles en Tabla 1).

Fig. 6.- Areas of endemicity, as determined by Wagner parsimony analysis. Sectors limited by thick lines (labelled I to X) are sets of quadrates characterized by at least two endemisms (I to X). Thin lines denote the geographical ranges of species or subspecies that are congruent with the most parsimonious area cladogram. Dotted lines denote sets of operative area units featured by bootstrap values higher than 66 in the consensus tree, but not characterized by any exclusive endemic taxon. Further details in Table 1. Note that three other areas of endemism (XI, XII, XIII) that were obtained by other means are not represented in the figure (see Table 1).

EL ANÁlisis DE PARSIMONIA COMO MÉTODO PARA LA DETECCIÓN DE ÁREAS DE CONCENTRACIÓN DE ENDEMISMOS

La aplicación del PAE-PCE permitió detectar diez áreas de endemicidad de las que cinco, constituidas por una única cuadrícula, eran evidentes. A pesar de los sucesivos análisis, los conjuntos de cuadrículas XI, XII y XIII (Tabla 1) no pudieron ser detectados por este medio, aunque no era difícil localizarlos por inspección visual de una matriz de similitudes. Esto se logró, de modo más directo y eficaz, con el método descrito por Luna Vega et al. (2000). Es evidente que una acumulación suficiente de especies de distribución incongruente con las agrupaciones de áreas más parsimoniosas, puede llegar a impedir la detección de áreas de endemicidad solapadas bajo el criterio de parsimonia más extendido. Esto resultará en agrupaciones estables no caracterizadas por especies exclusivas. En consecuencia, el método propuesto por García-Barros et al. (2002) no garantiza la identificación de todos los conjuntos de áreas de endemismo (tal como se definieron en la introducción). La misma idea, pero llevada a cabo con un método de construcción de cladogramas que impida la homoplasia, ofrece mayores garantías de obtener los resultados deseados. Es interesante que el sistema de Luna Vega et al. (2000) no fuese propuesto como un refinamiento del PAE, sino como un remedo de los análisis de compatibilidad para la definición de pistas generalizadas en panbiogeografía (o trazos generalizados: Craw, 1988). La presente experiencia demuestra que el principio de compatibilidad (Meacham \& Estabrook, 1985) es más adecuado para la detección de agregados de especies de igual distribución, siempre que se acepte la ya mencionada posibilidad de múltiples áreas de endemicidad con potencial solapamiento. En consecuencia, los programas de reconstrucción filogenética basados en el principio de compatibilidad pueden ser un sustituto útil de PAUP, si bien no siempre más cómodos o rápidos (por ejemplo CLIQUE del paquete PHYLIP: Felsenstein, 1985, o CLINCH: Fiala, 1984). Cabría mencionar, como alternativa a los métodos ya comentados, el pesado de los caracteres, basado en su propia consistencia (Farris, 1989) u otros criterios (Linder, 2001). No obstante, es dudoso que estos métodos permitan ahorrar tiempo para conseguir el objetivo mencionado. Por fín, aparte de todas estas consideraciones, los resultados pueden requerir, además, reconsideración intuitiva por cuanto se refiere a los grupos de especies de distribución "anidada" (anillos concéntricos) (Posadas \& Miranda-Esquivel, 1999; García-Barros et al., op. cit.); o bien, medidas probabilísticas de la fiabilidad de las asociaciones encontradas, punto que no se discutirá aquí (Szumik et al., 2002; Mast \& Nyffeler, 2003).

\section{EL PRINCIPIO DE PARSIMONIA COMO MÉTODO DE ASO- CIACIÓN}

Si se acepta que la presencia de dos o más especies exclusivas de idéntica distribución no es un criterio imprescindible para la delimitación de un área de interés para su posterior interpretación, la cons- 
Tabla 1.- Áreas de endemicidad identificadas mediante el análisis de parsimonia de Wagner (I-X) u otros métodos (XI-XIII). Los números romanos denotan áreas; las combinaciones de letras y números corresponden a las cuadrículas incluidas (dos números separados por un guión implican mención a las cuadrículas intermedias, p. ej. b9-11 significa b9, b10 y b11). La columna de la derecha recoge las especies y subespecies que caracterizan estas áreas. Ver Figura 6.

Table 1.- Areas of endemicity identified by means of Wagner parsimony (I-X) and other methods (XI-XIII). Roman numerals indicate the areas. Combinations of letters an numerals indicate single squares (two numbers connected by a hyphen imply a mention to all intermediate squares, e.g. b9-b11 means b9, b10 and b11). The species and subspecies that characterize these areas (synapocories) are given in the right column. See Figure 6.

\begin{tabular}{|c|c|c|}
\hline Área & Unidades & Taxones \\
\hline I & b3, c3-5, d4 & $\begin{array}{l}\text { Pseudochazara atlantis (Austaut, 1905) } \\
\text { Coenonympha vaucheri (Blachier, 1905) }\end{array}$ \\
\hline II & $\mathrm{c} 7, \mathrm{~d} 8$ & $\begin{array}{l}\text { Plebeius glandon (Prunner, 1798) zuellichi Hemming, } 1933 \\
\text { P. violetae (Gómez-Bustillo, Expósito \& Martínez, 1979) } \\
\text { P. golgus (Hübner, [1813]) } \\
\text { Melitaea athalia (Rottemburg, 1775) nevadensis Oberthür, } 1904 \\
\text { Arethusana arethusa (D. \& Schiff., 1775) boabdil (Rambur, [1840]) } \\
\text { Pseudochazara hippolyte (Esper, 1784) williamsi (Romei, 1927) }\end{array}$ \\
\hline III & d9-10 & $\begin{array}{l}\text { Polyommatus caelestissimus (Verity, 1921) } \\
\text { Erebia zapateri Oberthür, } 1875\end{array}$ \\
\hline IV & d11, e11-12 & $\begin{array}{l}\text { Erebia gorgone (Boisduval, [1833]) } \\
\text { E. lefebvrei (Boisduval, 1828) } \\
\text { E. sthennyo (Graslin, 1850) }\end{array}$ \\
\hline $\mathrm{V}$ & g13 & $\begin{array}{l}\text { Polyommatus humedasae (Toso \& Balletto, 1979) } \\
\text { Erebia christi (Rätzer, 1890) }\end{array}$ \\
\hline VI & h9-10 & $\begin{array}{l}\text { Papilio hospiton Genè, } 1839 \\
\text { Euchloe insularis (Staudinger, 1861) } \\
\text { Plebeius idas (L., 1761) bellieri Oberthür, 1910 } \\
\text { Aglais urticae (L., 1758) ichnusa (Hübner, 1824) } \\
\text { Argynnis elisa Godart, 1823 } \\
\text { Hipparchia aristaeus aristaeus (Bonelli, 1826) }\end{array}$ \\
\hline VII & h9 & $\begin{array}{l}\text { Pseudophilotes barbagiae de Prins \& van der Poorten, } 1982 \\
\text { Polyommatus coridon (Poda, 1761) gennargenti (Leigheb, 1987) } \\
\text { Maniola nurag (Ghiliani, 1852) }\end{array}$ \\
\hline VIII & i7 & $\begin{array}{l}\text { Cupido minimus (Fuessly, 1775) trinacariae Verity, } 1919 \\
\text { Melanargia occitanica (Esper, 1793) pherusa Boisduval, } 1833\end{array}$ \\
\hline IX & $\mathrm{m} 6$ & $\begin{array}{l}\text { Zerynthia cerisy Godart, } 1824 \text { cretica Rebel, } 1916 \\
\text { Plebeius psyloritus (Freyer, 1845) } \\
\text { Hipparchia cretica (Rebel, 1916) } \\
\text { Coenonympha thyrsis (Freyer, 1845) }\end{array}$ \\
\hline $\mathrm{X}$ & o6 & $\begin{array}{l}\text { Glaucopsyche melanops (Boisduval, [1828]) paphos Chapman, } 1920 \\
\text { Hipparchia cypriensis (Holik, 1949) } \\
\text { Maniola cypricola (Graves, 1928) }\end{array}$ \\
\hline XI & g12-13 & $\begin{array}{l}\text { Polyommatus exuberans (Verity, 1926) } \\
\text { Erebia aethiopella (Hoffmannssegg, 1806) }\end{array}$ \\
\hline XII & g13-14, h13-14 & $\begin{array}{l}\text { Erebia flavofasciata (Heyne, 1895) } \\
\text { E. tyndarus (Esper, 1781) }\end{array}$ \\
\hline XIII & b9-11, c9-11, d9-11, e10-11 & $\begin{array}{l}\text { Melitaea trivia (D. \& Schiff., 1775) ignasiti Sagarra, } 1926 \\
\text { Coenonympha glycerion (Borkhausen, 1788) iphioides (Staudinger, 1870) }\end{array}$ \\
\hline
\end{tabular}



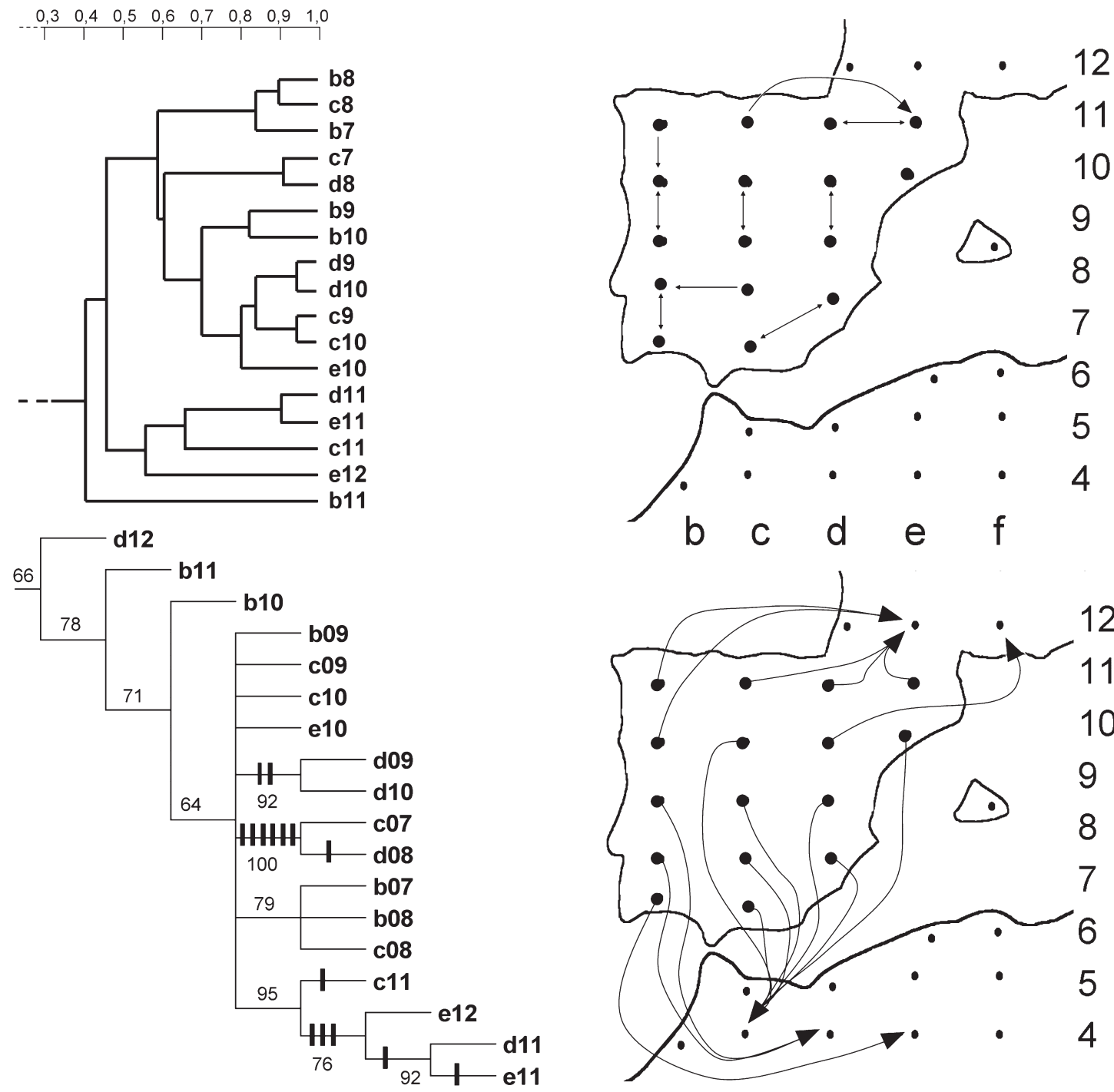

Fig. 7.- Relaciones entre las unidades de área de la Península Ibérica, y entre estas y su entorno geográfico inmediato. A la izquierda, porción del dendrograma de clasificación de todas las unidades de área, basado en el coeficiente de asociación de Jaccard y el método de aglomeración UPGMA, que incluye el terrirorio ibérico, y cladograma de áreas (las marcas negras representan especies exclusivas, y los números los valores de bootstrap). En los dos mapas de la derecha se muestran los máximos valores de asociación de cada unidad ibérica con cualquier otra (arriba), y el mayor valor con una unidad de área extraibérica (debajo). Cada flecha indica la dirección del coeficiente de asociación ( $J$, índice de Jaccard). No se representa el valor correspondiente a la isla de Mallorca, que la liga con la cuadrícula al en la costa occidental africana (tanto por el índice de asociación como en el análisis de parsimonia).

Fig. 7.- Relationships among the land squares belonging to the Iberian Peninsula, and between these and their immediate geographic context. Left, part of the general phenogram (Jaccard's index, UPGMA) relative to the circum-Iberian area, and area cladogram (black dots represent synapocories, endemic taxa exclusive to the clades denoted; the numerals indicate bootstrap values). The arrows in the two maps on the right side show the nearest neighbour for each Iberian land quadrat (above), and the nearest non-Iberian neighbour (below), both based on Jaccard's values. The links relative to the island of Mallorca (f9) are not represented; this island is primarily associated to square a1 on the western African coast (either on the basis of Jaccard index or based on parsimony analysis). 
trucción de dendrogramas de unidades de áreas por parsimonia de Wagner no es sino un método alternativo de clasificación jerárquica. Su principal inconveniente puede ser también su más evidente ventaja: en la práctica, el criterio de coincidencia exacta y congruencia entre las áreas de distribución es extraordinariamente restrictivo, y conduce a ignorar relaciones entre áreas de distribución "casicoincidentes" o solapadas. Efectos así tienden a reducir el peso de las especies de amplia distribución, menos informativas, lo que pudiera ser deseable en ocasiones. Esto se debe a la alta posibilidad de intersencción de los límites de las áreas de distribución de este tipo de taxones, al menos en algún punto, que es interpretado como incongruencia. En tanto que método de solución única (la más parsimoniosa), requeriría una medida de fiabilidad (referencias en Forey et al., 1992), pero este es un problema compartido con cualquier otro método de clasificación jerárquica (p. ej., Vargas \& Real, 1996; Vargas \& Palomo, 1997). Si se comparan todas las asociaciones marcadas en la Figura 6 (es decir, los clados obtenidos mediante parsimonia de Wagner, en un árbol de consenso estricto, y con índices de bootstrap superiores a 0,66 ), se observa una marcada similitud con los resultados de un dendrograma basado en el índice de Jaccard (Figura 4). La clasificación a grandes rasgos es, sin embargo, más estricta: en este caso concreto, desaparecen los clados basales apoyados en especies de distribución amplia. También desaparecen, necesariamente, las agrupaciones basadas en la ausencia de especies (por ejemplo, áreas 6, 7, 8, en la Figura 4), otra virtud del procedimiento. En la mayor parte de los casos en los que los resultados de este tipo se han comparado con los derivados del uso de índices de similitud, distancia o correlación, se ha constatado un nivel aceptable de concordancia general entre los resultados de ambos métodos (Posadas \& Miranda-Esquivel, 1999). En definitiva, el PAE es una alternativa potencialmente útil como método de clasificación, si bien poco flexible, y sensible a datos de baja calidad. Al emplearlo puede ser especialmente interesante realizar una estimación previa de la solidez de los datos de distribución, descartando unidades de área pobremente prospectadas, o fundiéndolas en unidades de mayor tamaño. Finalmente, como se ha visto, esta aplicación no es estrictamente equivalente -aunque pudiera parecerlo- a la identificación de áreas de endemicidad (sensu Morrone, 1994), en la que puede no ofrecer resultados satisfactorios. En tanto que procedimiento de asociación, cabría aquí recomendar el uso de la parsimonia de Wagner en lugar de com- patibilidad; en su caso, la homoplasia (dispersión o no) representa, en definitiva, afinidad entre conjuntos de unidades de área. El problema del soporte estadístico de los clados queda abierto a discusión, al igual que a propósito de la detección de áreas potenciales de endemicidad.

Sea cual fuere la puesta en práctica de la idea del PAE, es evidente que los métodos que requieren congruencia exacta son especialmente sensibles a los datos de baja fiabilidad (por ejemplo, muestreo insuficiente). Así lo sugiere el hecho de que produzca mejores resultados si se aplica a "áreas naturales" que a cuadrados arbitrarios (Morrone \& Escalante, 2002). En estas circusntancias puede ser especialmente interesante valorar, como primer paso, la calidad de los datos, o seleccionar las unidades de área que reunan mayores garantías para el estudio (p. ej. Hortal et al., 2001; Hortal \& Lobo, 2002; Hortal et al., en prensa).

ÁREAS DE CONCENTRACIÓN DE ENDEMISMOS, Y PATRONES DE DISTRIBUCIÓN DE LAS MARIPOSAS ENDÉMiCAS EN LA REGión PALEÁRTICA OCCIDENTAL

Destaca, en primer lugar, la concentración de elementos endémicos en las zonas montañosas de la periferia mediterránea, ya bien documentada (Dennis et al., 1991; Balletto, 1995; Dennis et al., 1995a, 1995b; Dennis \& Williams, 1995; Munguira, 1995), con especial densidad de endemismos de distribución restringida en el área alpina y en la Península Ibérica. Esta concentración, combinada con cierta proporción de especies y subespecies nórdicas, y una dominancia de endemismos de más amplia distribución en latitudes medias, determina un patrón con un marcado componente latitudinal en el norte de Europa, complicado en el sur por elementos de disposición transversal. Las agrupaciones basadas exclusivamente en endemismos (por ejemplo, Figuras 4 y 6) tienen mucho en común con los resultados apoyados en el conjunto de la fauna de ropalóceros (Dennis et al., 1991; Dennis \& Williams, 1995). De hecho, al menos como patrón general, parece existir una correlación positiva entre el número de endemismos y el número total de especies (Dennis et al., 1995, 1998), que aperece igualmente a nivel ibérico (García-Barros et al., 2000). Esta aparente coherencia es interesante desde un punto de vista práctico, ya que podría simplificar la caracterización estadística de grupos faunísticos y regiones faunísticas de ropalóceros en Europa occidental, encaminada a la selección de áreas de alta diversidad de mariposas.

Los resultados reflejan una disminución del tamaño del área de distribución de los endemismos 
en sentido nordeste-suroeste que, dada la correlación mencionada entre número de endemismos y diversidad faunística total, podría representar un fenómeno general entre la fauna de mariposas del paleártico occidental. Esto puede sugerir un "efecto Península" en Europa. O, quizá mejor, un "patrón península", término que permite soslayar los problemas de interpretación relativos a los mecanismos causantes (p. ej. Gutiérrez, 1997). Implica, en cualquier caso, una decreciente riqueza de especies desde el punto de contacto de un área peninsular con el continente, hacia su extremo más distante (p. ej. Blondel, 1979) como se observa, a menor escala, entre las mariposas ibéricas (Martín Cano, 1983; Martín \& Gurrea, 1990). No obstante, y más bien al contrario, la densidad de endemismos tiende a aumentar hacia el Oeste en el occidente paleártico. Como nota incidental, y dado que el gradiente de diversidad en Europa occidental parece contradecir este "patrón península", es razonable inducir que el gradiente observado en la Península Ibérica sea debido a la extinciones o desplazamientos de áreas de distribución en sentido sur-norte, consecutivas a fenómenos de dispersión post-glacial en el mismo sentido (como afirma Hewitt, 1999).

Pueden destacarse ciertos detalles de interés a relativos al territorio ibero-balear. En primer lugar la isla de Mallorca, desmarcada del territorio peninsular por su escasa diversidad de mariposas, y su casi completa carencia de endemismos, que hacen que esta isla contraste con otras de superficie comparable en el Mediterráneo. No obstante, al menos por cuanto se refiere al número total de especies, Mallorca parece seguir la tendencia general de las islas mediterráneas: el número de especies está negativamente correlacionado con su superficie y la distancia al continente (Hockin, 1980), en concordancia con las ideas de MacArthur \& Wilson (1967). En segundo lugar, puede sorprender la ausencia de un claro componente de afinidad norteafricana en Iberia. Tal componente existe, como se muestra en la Figura 7, afectando a las unidades ibéricas meridionales, pero es desbancado por el mayor peso de la similitud de las cuadrículas ibéricas entre ellas, y entre este conjunto y la fauna endémica pirenaica. Por fin, el área galaica, ligada al resto de la Península pero pobremente definida. Este puede ser un patrón propio de las mariposas diurnas, no compartido por otros grupos de organismos terrestres (p. ej. García-Barros et al., 2002). Pero tal vez esto refleja solo una parte de la realidad, si se tiene en cuenta la escasez de datos faunísticos detallados de las mariposas gallegas (García-Barros \& Munguira, 1999; García-Barros et al., 2000). De hecho, si consideramos las tendencias en diversidad de mariposas en la Península Ibérica (Martín \& Gurrea, 1990) o, más concretamente en su franja occidental (Garcia-Pereira, inédito; Hortal et al., en prensa), se refuerza la idea de que la fauna de Galicia y su periferia ha sido subestimada. Esta discusión conduce al problema de la evaluación de la información faunística previa al análisis biogeográfico, ya mencionado.

Como resumen hay que destacar, desde el punto de vista de la riqueza de endemismos de este grupo de insectos, la importancia de las áreas montañosas próximas al mediterráneo, en especial el Atlas, los Alpes y su zona de influencia. Y, tal vez de modo inesperado, en menor medida las situadas en las penínsulas del mediterráneo central y oriental. Hay que añadir las grandes islas mediterráneas: Córcega, Cerdeña, Sicilia, Creta y Chipre, pero no Mallorca. En la Península Ibérica se detectan tres áreas de concentración de mariposas endémicas bien diferenciadas, que corresponden a las cordilleras béticas, el Sistema Ibérico, y los Pirineos. Iberia descolla no solo por la densidad de especies, sino por la rareza relativa de sus elementos endémicos, y por el número de áreas de endemicidad independientes. Este territorio ha de ser interpretado más como área de yuxtaposición faunística que como "área de transición", en concordancia con otros sectores de la cuenca mediterránea (Balletto \& Casale, 1991), en parte consecuencia del papel de refugio de las penínsulas mediterráneas durante los periodos glaciales (Coope, 1990; De Jong, 1998; King \& Ferris, 1998; Taberlet et al., 1998; Schmitt \& Seitz, 2001; otros ejemplos en Hewitt, 1999). La circunstancia de área de refugio, combinada con el tamaño proporcionalmente reducido de las áreas de distribución de los endemismos suroccidentales, tiene relevantes implicaciones prácticas para el diseño de la preservación de la biodiversidad en Europa.

\section{AGRADECIMIENTOS}

Debo expresar mi agradecimiento a tres personas cuya revisión desinteresada y comentarios, además de los de los evaluadores, contribuyeron a mejorar el texto y depurar errores. Concretamente Joaquín Hortal, Paulas Posadas, y Patricio Pliscoff, incluso aunque no todos sus puntos de vista o sugerencias queden reflejados en el resultado. También a Jorge M. Lobo. Y con una dedicatoria especial a Fermín quien, sin embargo, ya a principios del Siglo XXI no tenía mucha confianza en el PAE: “¡Pero si esto ya no se hace!”. Parte de este trabajo fue financiado por el proyecto REN2000-0466/GLO. 


\section{Referencias}

BALletto, E., 1995. Endemism, areas of endemism, biodiversity and butterfly conservation in the EuroMediterranean area. Bollettino del Museo Regionale di Scienze Naturali - Torino, 13: 445-491.

Balletto, E. \& Casale, A., 1991. Mediterranean insect conservation. In: N.M. Collins \& J.A. Thomas (eds.). The conservation of insects and their habitats. Academic Press. London: 122-142.

Blondel, J., 1979. Biogéographie et écologie. Collection d'Écologie, 15. Masson. Paris.

Brooks, D. R. \& van Veller, M. G. P., 2003. Critique of parsimony analysis of endemicity as a method of historical biogeography. Journal of Biogeography, 30(3): 319-329.

BRUndin, L. Z., 1988. Phylogenetic biogeography. In: A. A. Myers \& P. S. Giller (eds.). Analytical biogeography. An integrated approach to the study of animal and plant distributions. Chapman \& Hall. London: 343-369.

Catena, A., Ramos, M. M. \& Trujillo, H. M., 2003. Análisis multivariado. Un manual para investigadores. Biblioteca Nueva. Madrid. 413 pp.

Coope, G. R., 1998. The invasion of Northern Europe during the Pleistocene by Mediterranean species of Coleoptera. In: F. Di Castri, A. J. Hansen \& M. De Bussche (eds.). Biological invasions in Europe and the Mediterranean Basin. Kluwer. Dordrecht: 203215.

CRAW, R. C., 1988. Continuing the synthesis between panbiogeography, phylogenetic systematics and geology as illustrated by empyrical studies on the biogeography of New Zealand and the Chatham Islands. Systematic Zoology, 37: 291-310.

Crisci, J., Lanteri, A. \& Ortiz-Jaureguízar, E., 1994. Programas de computación en sistemática y biogeografía histórica: revisión crítica y criterios para su selección. In: J. Llorente-Bousquets \& I. Luna Vega (compiladores). Taxonomía biológica. Fondo de Cultura Económica. México, D.F.: 207-225.

DA Silva \& OREN, D. C., 1996. Application of parsimony analysis of endemicity in Amazonian Biogeography: An example with Primates. Biological Journal of the Linnean Society, 39: 427-437.

DE JONG, H., 1998. In search of historical biogeographic patterns in the western Mediterranean terrestrial fauna. Biological Journal of the Linnnean Society, 65: 99-164.

Dennis, R. L. H., Shreeve, T. G. \& Williams, W. R., 1995a. Affinity gradients among European butterflies: evidence for an historical component to species distributions. Entomologist's Gazette, 46: 141-153.

Dennis, R. L. H.; Shreeve, T. G. \& Williams, W. R., 1995b. Taxonomic differentiation in species richness gradients among European butterflies (Papilionoi- dea, Hesperioidea): contribution of macroevolutionary dynamics. Ecography, 18: 27-40.

DENNIS, R. L. H. \& Williams, W. R., 1995. Implications of biogeographical structures for the conservation of European butterflies. In: A.S. Pullin (ed.). Ecology and conservation of butterflies. Chapman \& Hall. London: 213-229.

Dennis, R. L. H., Williams, W. R. \& Shreeve, T. G., 1991. A multivariate approach to the determination of faunal units among European butterfly species (Lepidoptera: Papilionoidea, Hesperioidea). Zoological Journal of the Linnean Society, 101: 1-49.

Dennis, R. L. H., Williams, W. R. \& Shreeve, T. G., 1998. Faunal structures among European butterflies: evolutionary implications of bias for geography, endemism and taxonomic affiliation. Ecography, 21: 181-203.

ENGHOFF, H., 2000. Reversals as branch support in biogeographical parsimony analysis. Vie et Milieu, 50: 255-260.

Espinosa, D., Aguilar, C. \& Escalante, T., 2001. Endemismo, áreas de endemismo y regionalización biogeográfica. In: J. Llorente Bousquets \& J. J. Morrone (eds.). In: Introducción a la Biogeografia en Latinoamérica: Teorías, conceptos, métodos y aplicaciones. Facultad de Ciencias, UNAM. México: 31-37.

Espinosa, D., Morrone, J. J., Llorente, J. \& Flores, O., 2002. Introducción al análisis de patrones en biogeografía histórica. Las Prensas de Ciencias, UNAM. México. 133 pp.

FARRIS, J. S., 1989. The retention index and the rescaled consistency index. Cladistics, 5: 417-419.

Felsenstein, J. F., 1995. PHYLIP, Phylogeny Inference Package, 3.57c. University of Washington. Seattle.

FialA, K., 1984. CLINCH. Cladistic Inference using Character Compatibility, 6.2. User's Manual. New York.

Forey, P. L., Humphries, C. J., Kitching, I. J., Scotland, R. W., Siebert, D. J. \& Williams, D. M., 1992. Cladistics. A practical course in systematics. Clarendon Press. Oxford. 191 pp.

García-Barros, E., García Pereira, P. \& Munguira, M. L., 2000. The geographic distribution and state of butterfly faunistic studies in Iberia (Lepidoptera, Papilionoidea, Hesperioidea). Belgian Journal of Entomolgy, 2: 111-124.

García-Barros, E., Gurrea, P., Luciáñez, M. J., Martín Cano, J., Munguira, M. L., Moreno, J. C., Sainz, H., SAnZ, M. J. \& Simón, J. C., 2002. Parsimony analysis of endemicity and its application to animal and plant distributions in the IberoBalearic region (western Mediterranean). Journal of Biogeography, 29: 109-124.

García-Barros, E. \& Munguira, M. L., 1999. Faunística de mariposas diurnas en España peninsu- 
lar. Áreas poco estudiadas: una evaluación en el umbral del Siglo XXI (Lepidoptera: Papilionidae \& Hesperiidae). SHILAP Revista de lepidopterología, 27(106): 189-202.

Garcia-Pereira, P. N. C., 2003. Mariposas diurnas de Portugal continental: Faunistica, biogeografía y conservación. Tesis Doctoral. Facultad de Ciencias, Universidad Autónoma de Madrid. Madrid. 371 pp. Inédito.

GERAADS, D., 1998. Biogeography of circumMediterranean Miocene-Pliocene rodents; a revision using factor analysis and parsimony analysis of endemicity. Palaeogeography, Palaeoclimatology, Palaeoecology, 137: 273-288.

Glasby, C. J. \& Alvarez, B., 1999. Distribution patterns and biogeographic analysis of Austral Polychaeta (Annelida). Journal of Biogeography, 26: 507-533.

GolobofF, P. A., 1993a. Estimating character weights during tree search. Cladistics, 9: 83-91.

Goloboff, P. A., 1993b. Nona, versión 2.0. Instituto Miguel Lillo. Tucumán.

GUTIÉRREZ, D., 1997. Importance of historical factors on species richness and composition of butterfly assemblages (Lep.: Rhopalocera) in a North Iberian mountain range. Journal of Biogeography, 24: 77-88.

Harold, A. S. \& Mooi, R. D., 1994. Areas of endemism: Definition and recognition criteria. Systematic Biology, 43: 438-411.

Hausdorf, B., 2002. Units in biogeography. Systematic Biology, 51: 648-652.

Hesselbarth, G., van Oorschot, H. \& Wagener, S., 1995. Die Tagfalter der Tüekei. Vol. 3, Tafeln und Verbreitungskarten. Fruckhaus Cramer. Greven. 847 pp.

Hewitt, G. M., 1999. Post-glacial re-colonization of European biota. Biological Journal of the Linnean Society, 68: 87-112.

Higgins, L. G. \& Hargraves, B., 1983. A field guide to the butterflies of Britain and Europe. Collins, London. $254 \mathrm{pp}$.

Higgins, L. G. \& RiLey, N. D., 1980. Guía de campo de las mariposas de España y Europa. Omega. Barcelona. $452 \mathrm{pp}$.

HockIN, D. C., 1980. The biogeography of the Mediterranean Islands. Nota lepidopterologica, 3: $119-125$

Hortal, J., García-Pereira, P. \& García-Barros, E., en prensa. Butterfly species richness in mainland Portugal. Geographic distribution patterns and conservation planning for predictive models. Ecography.

Hortal, J. \& LobO, J. M., 2002. Una metodología para predecir la distribución espacial de la diversidad biológica. Ecología (n.s.), 16: 151-178.
Hortal, J., Lobo, J. M. \& Martín-Piera, F., 2001. Forecasting insect species richness scores in poorly surveyed territories: the case of Portuguese dung beetles (Col. Scarabaeinae). Biodiversity and Conservation, 10: 1343-1367.

KInG, R. A. \& Ferris, C., 1998. Chloroplast DNA phylogeography of Alnus glutinosa (L.) Gaertn. Molecular Ecology, 7: 1157-1161.

Kostrowicki, A. S., 1969. Geography of the Palaearctic Papilionoidea (Lepidoptera). Panstwowe Wydawnictwo Naukowe. Krakow. 380 pp.

KuDRnA, O., 2002. The distribution atlas of European butterflies. Oedippus, 20: 1-342.

Linder, H. P., 2001. On areas of endemism, with an example from the African Restionaceae. Systematic Biology, 50(6): 892-912.

Llorente Bousquets, J. \& Morrone, J. J. (eds.), 2001. Introducción a la biogeografía en Latinoamérica: teorías, conceptos, métodos y aplicaciones. Las Prensas de Ciencias. UNAM. México. 277 pp.

Luna Vega, I., Alcántara Ayala, O., Morrone, J. J. \& EsPinOSA ORganista, D., 2000. Track analysis and conservation priorities in the cloud forests of Hidalgo, Mexico. Diversity and Distributions, 6: 137-143.

MacArthur, R. H. \& Wilson, E. O., 1967. The theory of island biogeography. Monographs in Population Biology, 1. Princeton University Press. Princeton. 203 pp.

MAJOR, J., 1988. Endemism: a botanical perspective. In: A. A. Myers \& P. S. Giller (eds.). Analytical biogeography. An integrated approach to the study of animal and plant distributions. Chapman \& Hall. London: 117-146.

Martín Cano, J., 1983. Species densities and geographical distribution of Lycaenids on the Iberian Peninsula. Nota lepidopterologica, 6(1): 24-34.

Martín, J. \& Gurrea, P., 1990. The peninsular effect in Iberian butterflies (Lepidoptera: Papilionoidea and Hesperioidea). Journal of Biogeography, 17: 85-96.

Martín-Piera, F. \& SANMARTín, I., 1999. Biogeografía de áreas y biogeografía de artrópodos holárticos y mediterráneos. Boletín de la Sociedad Entomológica Aragonesa, 26: 535-560.

Mast, A. R. \& Nyffeler, R., 2003. Using a null model to recognize significant co-occurence prior to identifying candidate areas of endemism. Systematic Biology, 52(2): 271-280.

Meacham, C. A. \& Estabrook, G. F., 1985. Compatibility methods in systematics. Annual Review of Ecology and Systematics, 16: 431-446.

Morrone, J. J., 1994. On the identification of areas of endemism. Systematic Biology, 43: 438-441.

Morrone, J. J. \& Crisci, J. V., 1995. Historical biogeography: Introduction to methods. Annual Review of Ecology and Systematics, 26: 373-401. 
Morrone, J. J. \& Escalante, T., 2002. Parsimony Analysis of Endemicity (PAE) of Mexican terrestrial mammals at different units: when size matters. Journal of Biogeography, 29(8): 1095-1104.

MunguiRA, M. L., 1995. Conservation of butterfly habitats and diversity in European Mediterranean countries. In: A. S. Pullin (ed.). Ecology and conservation of butterflies. Chapman \& Hall. London: 277-289.

Myers, A. A., 1991. How did Hawaii accumulate its biota? A test from the Amphipoda. Global Ecology and Biogeography letters, 1: 24-29.

Nel, A., Nel, J., Masselot, G. \& Thomas, A., 1998. An investigation into the application of the Wagner parsimony method in synecology. Biological Journal of the Linnean Society, 65: 165-189.

Nelson, G. \& Platnick, N., 1981. Systematics and biogeography: Cladistics and vicariance. Columbia University Press. New York. 567 pp.

NIXON, K. C., 1999a. Winclada, version BETA 0.9.92. University of Ithaca. New York.

NixON, K. C., 1999b. The parsimony Ratchet, a new method for rapid parsimony analysis. Cladistics, 15: 407-414.

Posadas, P., Estévez, J. M. \& Morrone, J. J., 1997. Distributional patterns and endemism areas of vascular plants in the Andean subregion. Fontqueria, 48: 110.

Posadas, P. \& Miranda-Esquivel, D. R., 1999. El PAE (Parsimony Analysis of Endemicity) como una herramienta en la evaluación de la biodiversidad. Revista Chilena de Historia Natural, 72: 539-546.

REAL, R. \& VARGAS, J. M., 1996. The probabilistic basis of Jaccard's index of similarity. Systematic Biology, 45: $380-385$.

Rojas-Soto, O. R.; Alcántara-Ayala, O. \& NaVArro, A. G., 2003. Regionalization of the avifauna of the Baja California Peninsula, Mexico: a parsimony analysis of endemicity and distributional modelling approach. Journal of Biogeography, 30(3): 449-461.

Rosen, B. R., 1988. From fossils to earth history: applied historical biogeography. In: A. A. Myers \& P. S. Giller (eds.). Analytical biogeography: An integrated approach to the study of animal and plant distributions. Chapman \& Hall. London \& New York: 437-481.

Schmitt, T. \& SeITz, A., 2001. Allozyme variation in Polyommatus coridon (Lepidoptera: Lycaenidae): identification of ice-age refugia and reconstruction of post-glacial expansion. Journal of Biogeography, 28: $1129-1136$.

SPSS, INC., 2001. SPSS para Windows. SPSS Inc. Chicago.

StatSoft, Inc., 1996. Statistica for Windows, Release 5.1 (computer program manual). Tulsa, Oklahoma.

SwOFFORD, D. L., 2002. PAUP (and other methods). Phylogenetic Analysis Using Parsimony. Version 4 beta. Sinauer Associates. Sunderland, Massachussetts.

Szumik, C. A., Cuezzo, F., Goloboff, P. A. \& Chalup, A., 2002. An optimality cryterion to determine areas of endemism. Systematic Biology, 51(5): 806-816.

Taberlet, P., Fumagalli, L., Wust-Saucy, A. G. \& Cossons, J. F., 1998. Comparative phylogeograohy and post-glacial colonization routes in Europe. Molecular Ecology, 7: 453-464.

Tennent, J., 1996. The butterflies of Morocco, Algeria and Tunisia. Gem Publishing Company. Wallingford. $217 \mathrm{pp}$.

Tolman, T. \& Lewington, R., 1997. Butterflies of Britain and Europe. HarperCollins Publishers. London. 320 pp.

Trejo-Torres, C. \& ACkerman, J. D., 2001. Biogeography of the Antilles based on a parsimony analysis of orchid distributions. Journal of Biogeography, 28: 775-794.

Vargas, J. M. \& Palomo, L. J., 1997. On identifying significant co-occurrence of species in space and time. Miscel·lània Zoològica, 20: 49-58.

Watanabe, K., 1998. Parsimony analysis of the distribution patterns of Japanese primary freshwater fishes, and its application to the distribution of the bagrid catfishes. Ichthyological Research, 45(3): 259-270. 
Apéndice.- Datos de presencia (1) o ausencia (0) de las especies o subespecies de mariposas endémicas. Las unidades de área operativas se denotan mediante un sistema de una letra (eje horizontal) y un número (eje vertical), ver Figura 1. Los taxones (columnas) están numerados (1 a 196), de acuerdo con la siguiente equivalencia:

Appendix.- Presence (1) and absence (0) data of the endemic species and subespecies of butterflies. The operative area units
are denoted by means of one letter (horizontal axis) and one number (vertical axis). The taxa (columns) are numbered (1 to 196) according to the following list:

$\mathbf{1}=$ Papilio hospiton Guenée, 1839

$\mathbf{2}=$ Parnassius phoebus sacerdos Stichel, 1906

$3=$ Zerynthia cerisy cretica (Rebel, 1904)

4= Zerynthia rumina (Linnaeus, 1758)

5= Pieris balcana Lorkovic, 1970

$\mathbf{6}=$ Anthocharis belia $($ L., 1767)

7= Anthocharis euphenoides Staudinger, 1869

$\mathbf{8}=$ Euchloe crameri Butler, 1869

$9=$ Euchloe insularis (Staudinger, 1861)

$\mathbf{1 0}=$ Euchloe simplonia $($ Boisduval, 1828)

$\mathbf{1 1}=$ Euchloe tagis (Hübner, 1804)

$\mathbf{1 2}=$ Zegris eupheme meridionalis Lederer, 1852

$\mathbf{1 3}=$ Colias phicomone (Esper, 1780)

14= Colias nastes werdandi Zetterstedt, 1840

$\mathbf{1 5}=$ Colias aurorina heldreichii Staudinger, 1862

16= Colias hecla sulitelma Aurivillius, 1890

$\mathbf{1 7}=$ Colias caucasica balcanica Rebel, 1901

18= Callophrys avis Chapman, 1909

19= Satyrium esculi (Hübner, 1804)

$\mathbf{2 0}=$ Tomares ballus (Fabricius, 1787)

$\mathbf{2 1}=$ Tomares mauretanicus (Lucas, 1849)

22= Cigaritis allardi Oberthür, 1909

23= Cigaritis siphax (Lucas, 1849)

24= Cigaritis zohra Donzel, 1847

$\mathbf{2 5}=$ Laeosopis roboris (Esper, 1793)

26= Lycaena phlaeas polaris Courvoisier, 1911

$\mathbf{2 7}=$ Lycaena dispar dispar (Haworth, 1802)

$\mathbf{2 8}=$ Lycaena ottomana $($ Lefèbvre, 1830$)$

29= Lycaena tityrus bleusei Oberthür, 1884

$\mathbf{3 0}=$ Lycaena alciphron gordius (Sulzer, 1776)

31= Lycaena alciphron heracleana Blachier, 1908

32 = Lycaena phoebus (Blachier, 1905)

33= Pseudophilotes barbagiae De Prins \& Poorten, 1982

34 $=$ Pseudophilotes baton (Bergsträsser, 1779)

35= Pseudophilotes panoptes (Hübner, [1813])

36= Cupido carswelli Stempffer, 1927

37 $=$ Cupido lorquinii (Herrich-Schäffer, 1847)

38= Cupido minimus trinacariae (Verity, 1919)

39= Turanana endymion taygetica (Rebel, 1902)

40= Maurus vogelii (Oberthür, 1920)

41= Glaucopsyche melanops (Boisduval, 1828)

$\mathbf{4 2}=$ Glaucopsyche paphos Chapman, 1920

43= Plebeius pylaon hespericus (Rambur, 1839)

$\mathbf{4 4}=$ Plebeius pylaon trappi (Verity, 1927)

45=Plebeius argus caernensis Thompson, 1941

46= Plebeius bellieri (Oberthür, 1910)

47 $=$ Plebeius martini martini (Allard, 1867)

$\mathbf{4 8}=$ Plebeius psyloritus (Freyer, 1845)

$49=$ Plebeius martini ungemachi (Rothschild, 1926)

$\mathbf{5 0}=$ Plebeius glandon aquilo (Boisduval, 1832)

$\mathbf{5 1}=$ Plebeius glandon zuellichi (Hemming, 1933)

$\mathbf{5 2}=$ Plebeius pyrenaicus asturiensis (Oberthür, 1910)

$\mathbf{5 3}=$ Plebeius pyrenaicus pyrenaicus (Boisduval, 1840)

54= Plebeius cramera $($ Eschscholtz, 1821)

$\mathbf{5 5}=$ Plebeius morronensis Ribbe, 1910

56 $=$ Plebeius hyacinthus (Herrich-Schäffer, [1847])

$\mathbf{5 7}=$ Plebeius montensis (Verity, 1928)

$\mathbf{5 8}=$ Polyommatus dolus (Hübner, 1823)

$\mathbf{5 9}=$ Polyommatus ainsae (Forster, 1961)

$\mathbf{6 0}=$ Polyommatus aroaniensis (Brown, 1976)

61 $=$ Polyommatus fabressei Oberthür, 1910

62= Polyommatus humedasae Toso \& Balletto, 1976

63= Polyommatus exuberans (Verity, 1926)

64= Polyommatus galloi Balletto \& Toso, 1979

65 = Polyommatus nephohyptamenus (Brown \& Coutsis, 1978)

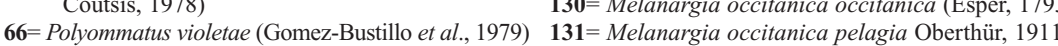

$\mathbf{6 7}=$ Polyommatus sertavulensis $($ Koçak, 1979)

$\mathbf{6 9}=$ Polyommatus golgus (Hübner, 1813)

$\mathbf{7 0}=$ Polyommatus nivescens Keferstein, 185

$\mathbf{7 1}=$ Polyommatus escheri (Hübner, 1823)

$\mathbf{7 2}=$ Polyommatus coridon gennargenti (Leigheb, 1987)

73= Polyommatus caelestissimus Verity, 1921

$\mathbf{7 4}=$ Polyommatus albicans (Herrich-Schäffer, 1851)

75 $=$ Polyommatus hispanus (Herrich-Schäffer, 1852)

76 $=$ Polyommatus punctifera (Oberthür, 1876)

$77=$ Aglais urticae ichnusa $($ Hübner, 1824)

$\mathbf{7 8}=$ Euphydryas beckeri (Lederer, 1853)

79= Euphydryas debilis (Oberthür, 1909)

$\mathbf{8 0}=$ Euphydryas aurinia glaciegenita (Verity, 1928)

81 = Euphydryas cynthia $($ Denis \& Schiffermüller, 1775)

$\mathbf{8 2}=$ Euphydryas desfontainii baetica (Rambur, 1858)

$\mathbf{8 3}=$ Euphydryas desfontainii desfontainii (Godart, 1819)

$\mathbf{8 4}=$ Euphydryas intermedia wolfensbergeri (Frey, 1880)

$\mathbf{8 5}=$ Melitaea aetherie (Hübner, 1826)

$\mathbf{8 6}=$ Melitaea asteria Freyer, 1828

$\mathbf{8 7}=$ Melitaea deione (Geyer, 1832)

$\mathbf{8 8}=$ Melitaea nevadensis Oberthür, 1904

89= Melitaea parthenoides Keferstein, 1851

$\mathbf{9 0}=$ Melitaea trivia ignasiti Sagarra, 1926

91 = Melitaea varia Meyer-Dür, 1851

92 = Argynnis elisa Godart, 1823

93 = Fabriciana adippe auresiana (Fruhstorfer, 1908)

$\mathbf{9 4}=$ Boloria eunomia ossiana (Herbst, 1800)

96= Boloria graeca (Staudinger, 1870)

97= Boloria improba improbula $($ Bryk, 1920)

$\mathbf{9 8}=$ Satyrus actaea (Esper, [1780])

$9=$ Satyrus ferula atlantea (Verity, 1927)

$\mathbf{1 0 0}=$ Berberia abdelkader (Pierret, 1837)

101= Oeneis glacialis (Moll, 1783)

102 = Arethusana arethusa boabdil (Rambur, [1840])

103 $=$ Chazara prieuri (Pierret, 1837)

104= Pseudochazara atlantis (Austaut, 1905)

$\mathbf{1 0 5}=$ Pseudochazara graeca (Staudinger, 1870)

106= Pseudochazara hippolyte williamsi Romei, 1927

$\mathbf{1 0 7}=$ Pseudochazara lydia lydia (Staudinger, 1878)

108= Pseudochazara mamurra amymone Brown, 1976

109= Pseudochazara orestes De Prins \& Poorten, 1981

$110=$ Pseudochazara mniszechii tisiphone Brown, 1980

111 $=$ Pseudochazara cingovskii Gross, 1973

112 = Pseudochazara anthelea amalthea (Frivaldsky, 1845)

$113=$ Hipparchia alcyone alcyone (Denis \& Schiffermüller, 1775)

114= Hipparchia alcyone caroli (Rothschild, 1933)

115= Hipparchia ellena (Oberthür, 1894)

16= Hipparchia neomiris (Godart, 1822)

$117=$ Hipparchia volgensis delattini Kudrna, 1975

$118=$ Hipparchia semele (Linnaeus, 1758)

119= Hipparchia cretica (Rebel, 1916)

120 = Hipparchia cypriensis Holik, 1949

121 = Hipparchia aristaeus aristaeus (Bonelli, 1826)

122= Hipparchia aristaeus senthes (Fruhstorfer, 1908)

123 = Hipparchia algirica (Oberthür, 1876)

124= Hipparchia aristaeus blachieri (Frühstorfer, 1908)

125= Hipparchia mersina (Staudinger, 1871)

126= Hipparchia hansii (Austaut, 1879)

127= Hipparchia powelli (Oberthür, 1910)

28 = Hipparchia fidia (Linnaeus, 1767)
68= Polyommatus atlanticus (Eversmann, 1843)

95= Boloria pales (Denis \& Schiffermüller, 1775)
132= Melanargia occitanica pherusa (Boisduval, 1833)

133 $=$ Melanargia arge (Sulzer, 1776)

134= Melanargia ines $($ Hoffmannsegg, 1804)

135= Melanargia galathea lucasi (Rambur, 1858)

136= Erebia eriphyle (Freyer, 1836)

137 $=$ Erebia manto $($ Denis \& Schiffermüller, 1775)

138= Erebia serotina Descimon \& de Lesse, 1953

139= Erebia claudina (Borkhausen, 1789)

$\mathbf{1 4 0}=$ Erebia flavofasciata Heyne, 1895

141 $=$ Erebia epiphron $($ Knoch, 1783)

142 $=$ Erebia orientalis Elwes, 1909

$\mathbf{1 4 3}=$ Erebia christ $i$ Rätzer, 1890

144 $=$ Erebia melampus (Fuessly, 1775)

$\mathbf{1 4 5}=$ Erebia pharte (Hübner, 1804)

146= Erebia sudetica sudetica Staudinger, 1861

147= Erebia sudetica liorana de Lesse, 1947

148 $=$ Erebia triaria $($ Prunner, 1798$)$

149 $=$ Erebia alberganus $($ Prunner, 1798)

$\mathbf{1 5 0}=$ Erebia pluto (Prunner, 1798)

$\mathbf{1 5 1}=$ Erebia gorge $($ Hübner, 1804)

152 $=$ Erebia aethiopella (Hoffmannsegg, 1806)

153 $=$ Erebia rhodopensis Nicholl, 1900

154 $=$ Erebia mnestra (Hübner, 1804)

$\mathbf{1 5 5}=$ Erebia gorgone Boisduval, 1833

156 = Erebia epistygne (Hübner, 1824)

$157=$ Erebia tyndarus $($ Esper, 1781)

158 $=$ Erebia cassioides (Reiner \& Hochenwarth, 1792)

159= Erebia arvenensis Oberthür, 1908

$\mathbf{1 6 0}=$ Erebia hispania Butler, 1868

161 = Erebia nivalis Lorkovic \& Lesse, 1954

162 $=$ Erebia calcaria Lorkovic, 1949

$163=$ Erebia ottomana Herrich-Schäffer, 1847

$\mathbf{1 6 4}=$ Erebia pronoe $($ Esper, 1780$)$

$165=$ Erebia melas (Herbst, 1796$)$

166 $=$ Erebia lefebvrei $($ Boisduval, 1828)

$\mathbf{1 6 7}=$ Erebia scipio $($ Boisduval, 1832)

$\mathbf{1 6 8}=$ Erebia styria $($ Godart, 1824)

169 $=$ Erebia styx $($ Freyer, 1834)

$170=$ Erebia montana $($ Prunner, 1798)

171 $=$ Erebia zapateri Oberthür, 1875

$\mathbf{1 7 2}=$ Erebia neoridas $($ Boisduval, 1828)

173 $=$ Erebia oeme $($ Hübner, 1804$)$

174= Erebia meolans (Prunner, 1798)

175 $=$ Erebia palarica Chapman, 1905

$\mathbf{1 7 6}=$ Erebia sthennyo Graslin, 1850

$\mathbf{1 7 7}=$ Coenonympha rhodopensis Elwes, 1900

$\mathbf{1 7 8}=$ Coenonympha thyrsis (Freyer, 1845)

179= Coenonympha corinna $($ Hübner, 1804)

$\mathbf{1 8 0}=$ Coenonympha dorus $($ Esper, 1782)

181 = Coenonympha vaucheri Blachier, 1905

182 $=$ Coenonympha gardetta $($ Prunner, 1798)

$\mathbf{1 8 3}=$ Coenonympha arcanioides (Pierret, 1837)

184 $=$ Coenonympha iphioides Staudinger, 1870

185= Maniola megala (Oberthür, 1909)

186= Maniola chia Thomson, 1987

$\mathbf{1 8 7}=$ Maniola nurag Ghiliani, 1852

188= Maniola halicarnassus Thomson, 1990

189= Maniola cypricola Graves, 1928

$\mathbf{1 9 0}=$ Hyponephele maroccana $($ Blachier, 1908)

191= Pyronia bathseba (Fabricius, 1793)

192 = Pyronia cecilia (Vallantin, 1894)

193= Pyronia tithonus (Linnaeus, 1767)

194= Pyronia janiriodes (Herrich-Schäffer, 1851)

$195=$ Lasiommata megera paramegaera (Hübner, 1824)

196= Lasiommata maera meadowaldoi (Rothschild, 1917). 


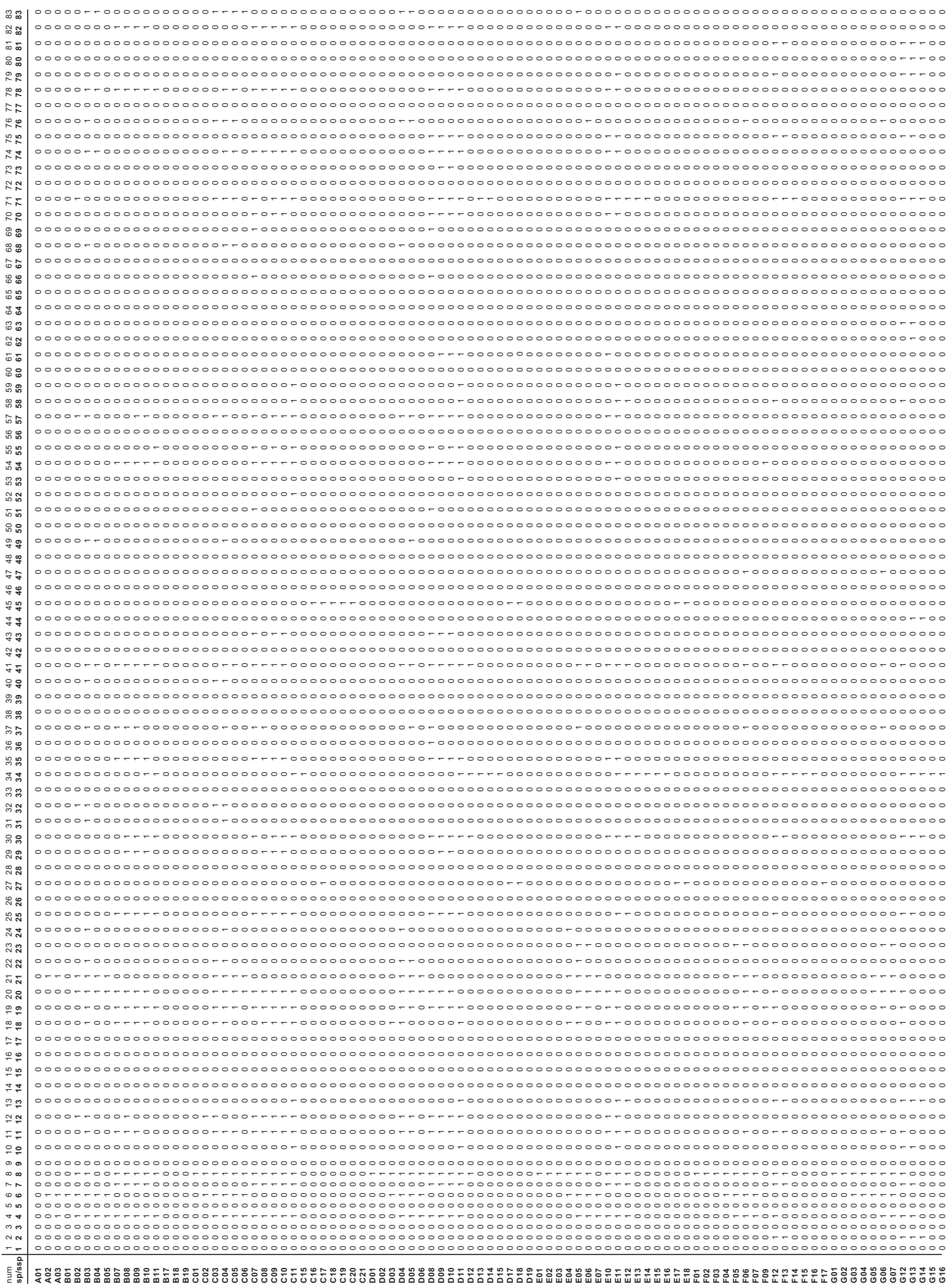




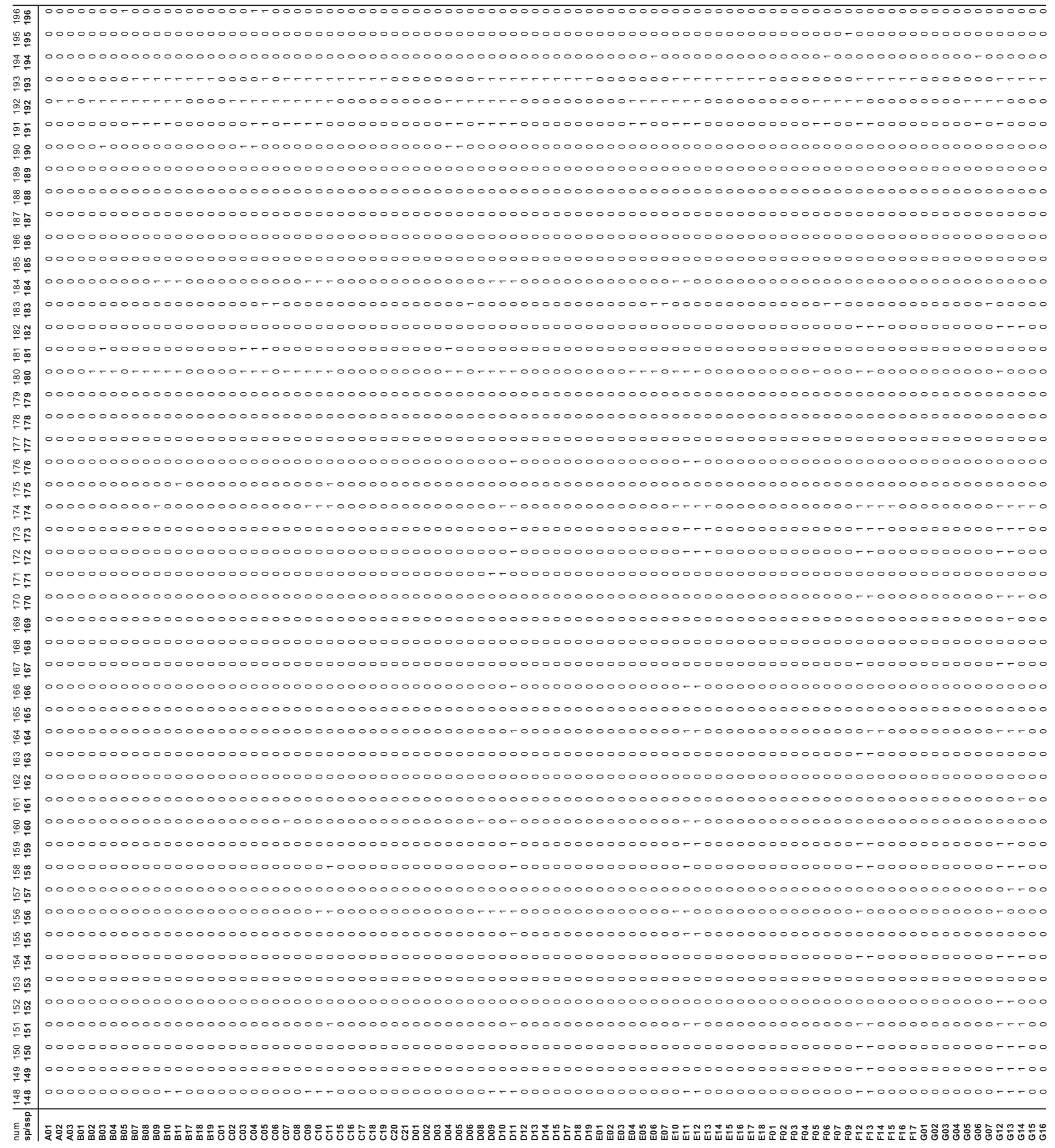




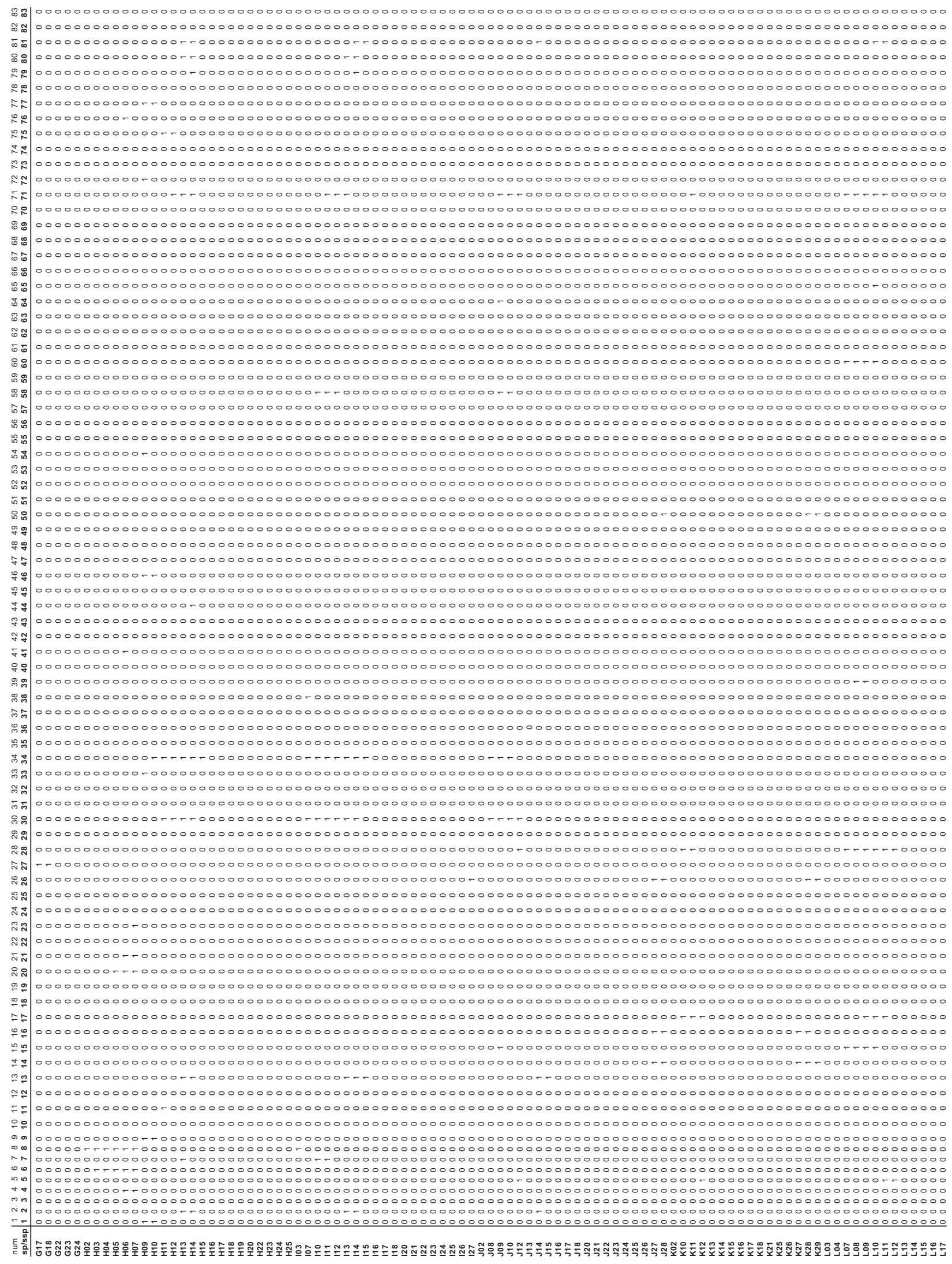




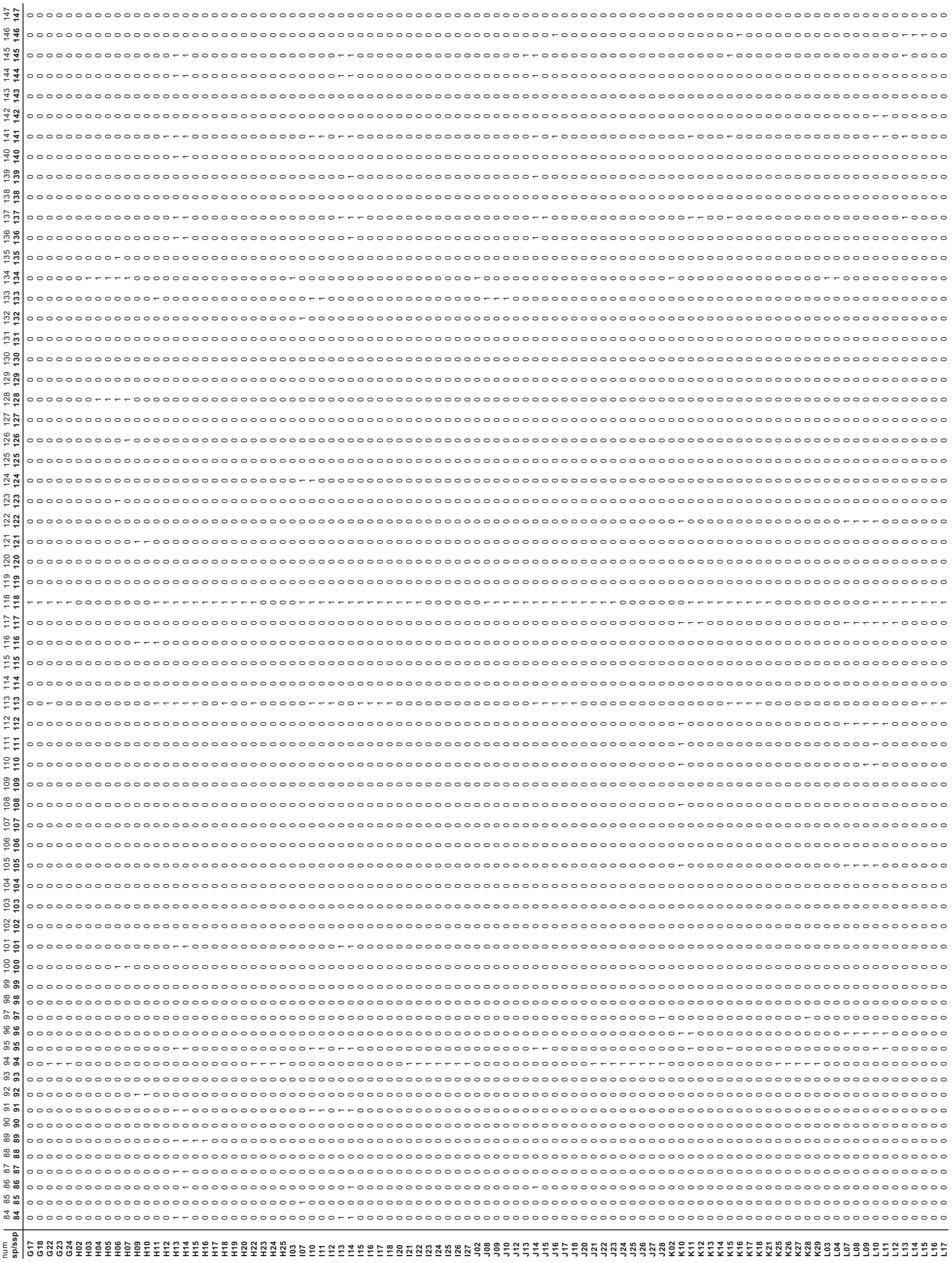




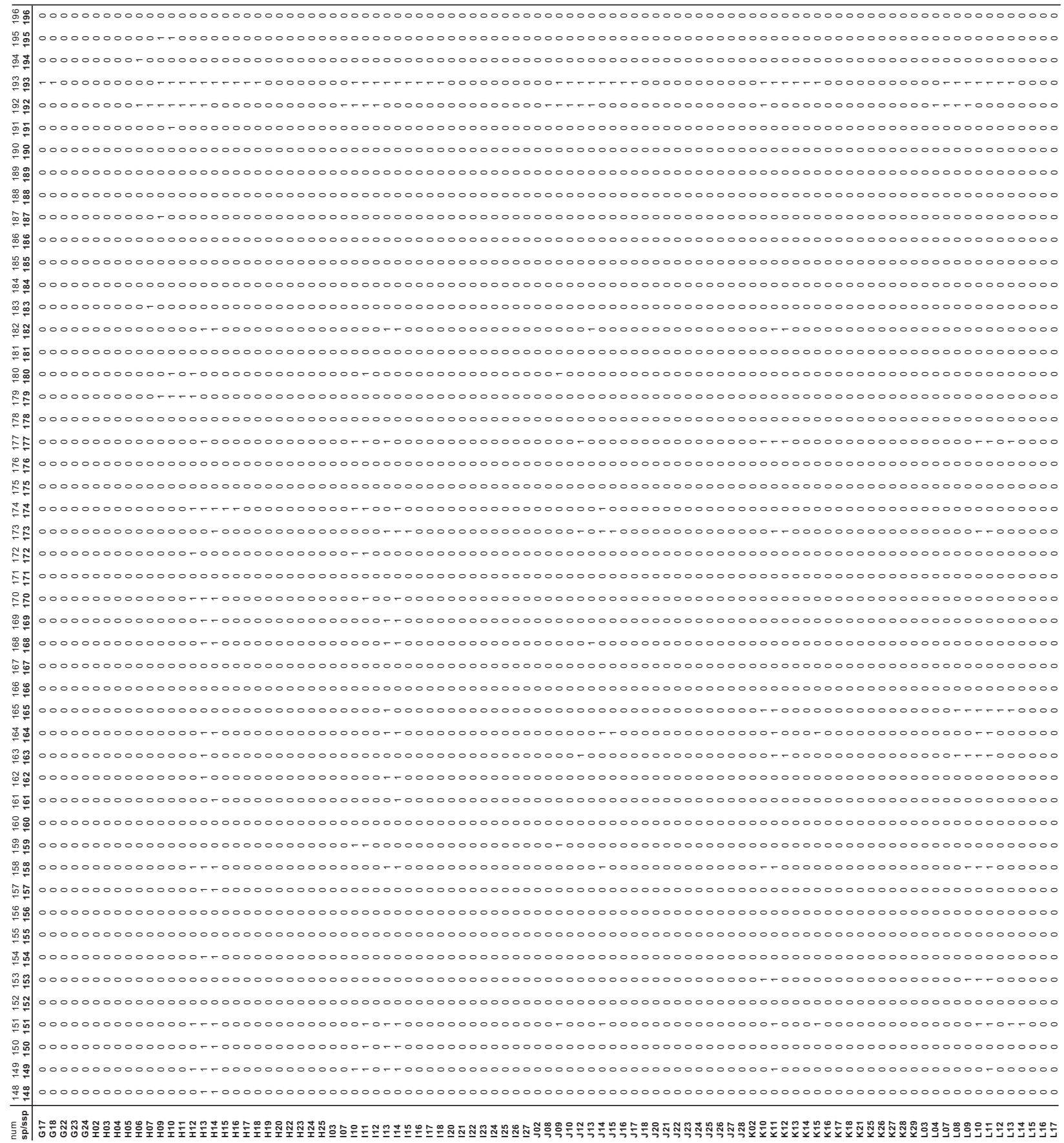




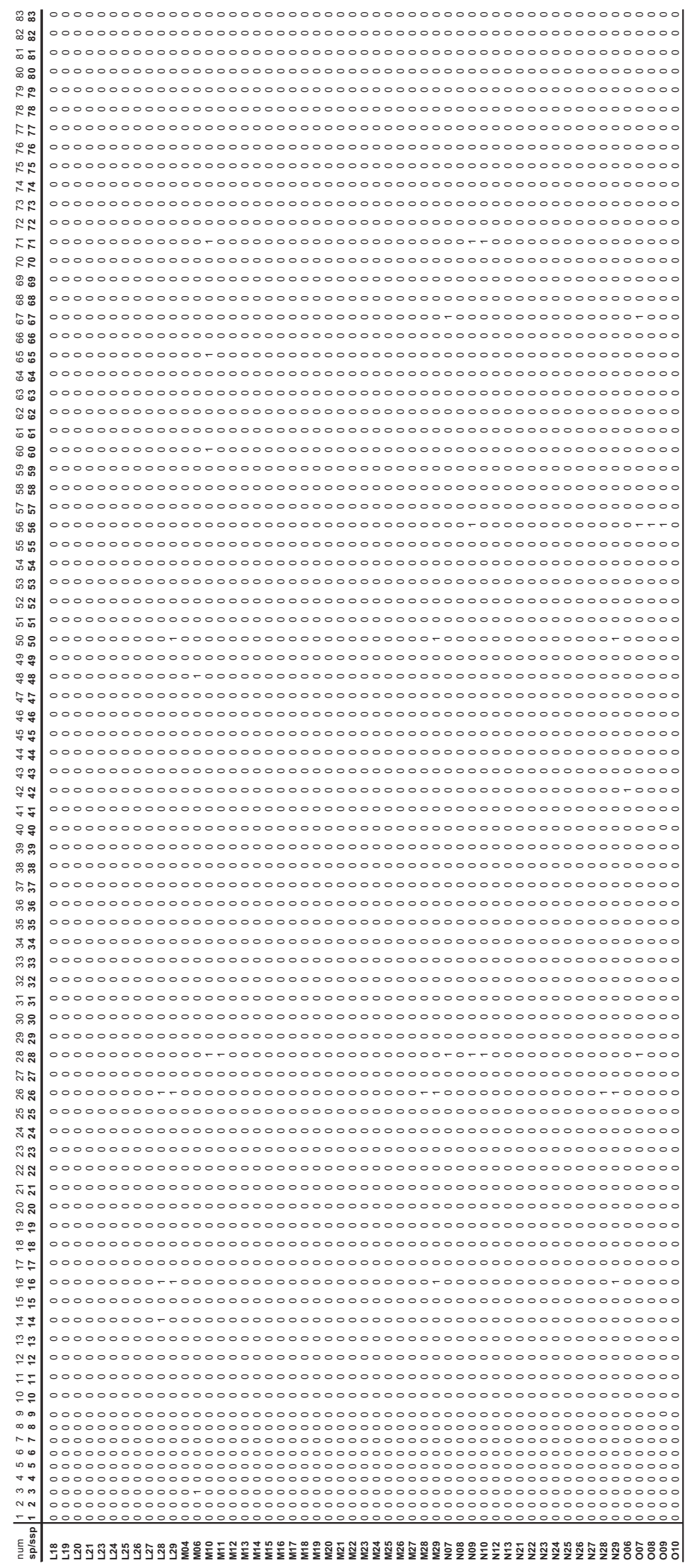




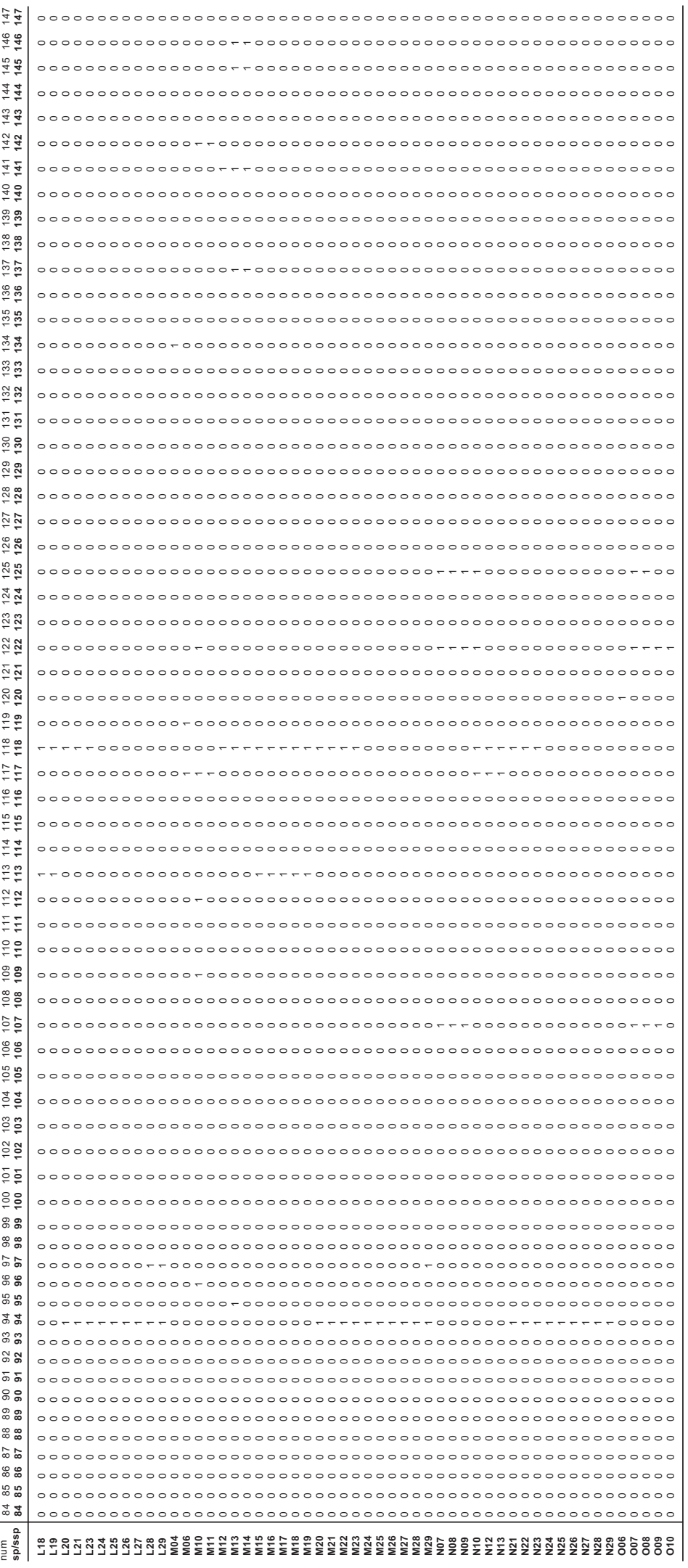

(c) Sociedad de Amigos del Museo Nacional de Ciencias Naturales y

Consejo Superior de Investigaciones Científicas Licencia Creative Commons 3.0 España (by-nc) http://graellsia.revistas.csic.es 


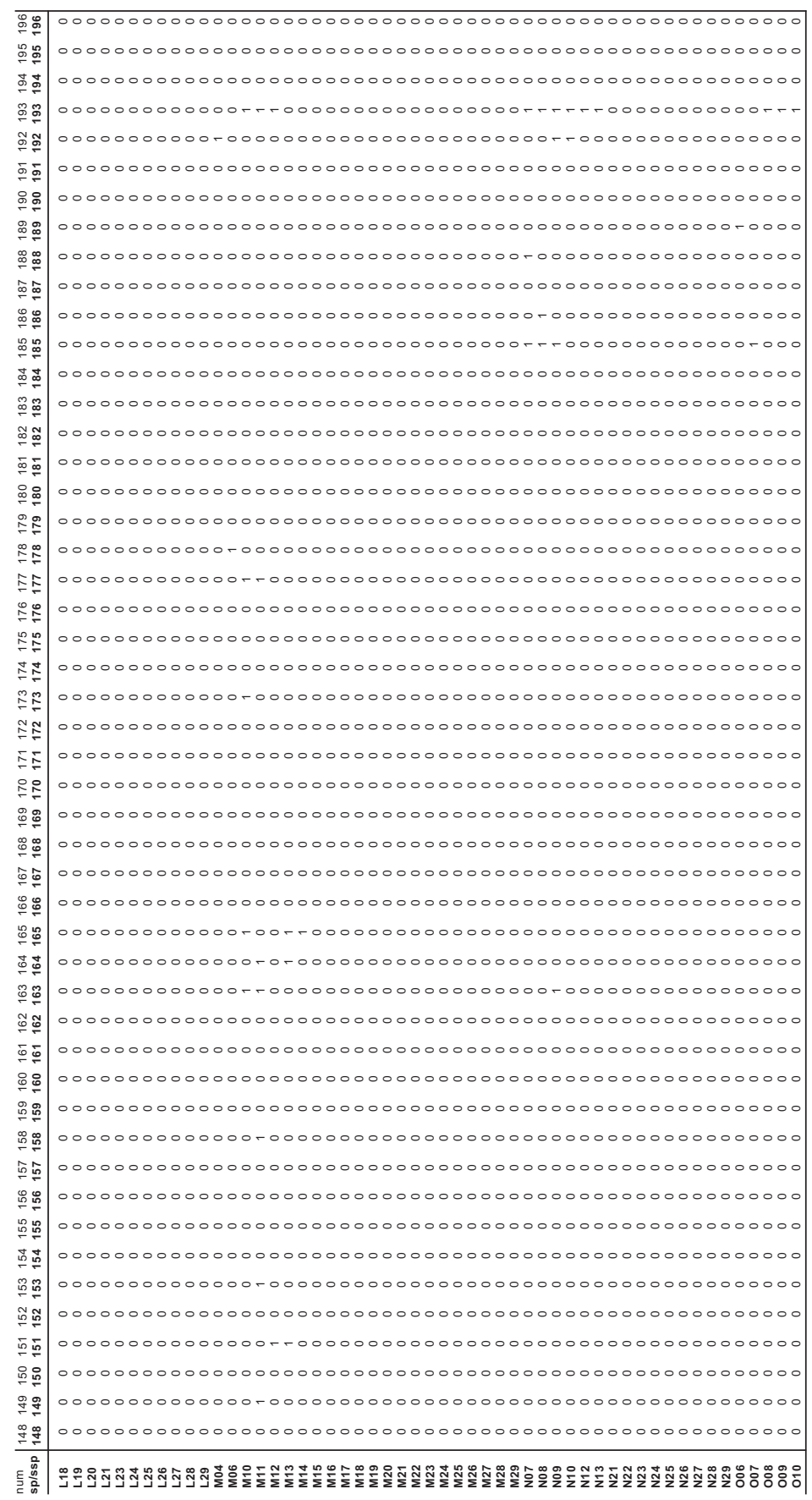

